\title{
A deep multi-band investigation of IC 2391 ${ }^{\star}$
}

\author{
L. Spezzi ${ }^{1,2}$, I. Pagano ${ }^{1}$, G. Marino ${ }^{1}$, G. Leto ${ }^{1}$, E. Young ${ }^{3}$, N. Siegler ${ }^{4}$, Z. Balog ${ }^{3}$, S. Messina ${ }^{1}$, E. Distefano ${ }^{1}$, \\ B. Merín ${ }^{5}$, and D. Barrado y Navascués ${ }^{6}$ \\ 1 INAF - Osservatorio Astrofisico di Catania, via S. Sofia 78, 95123 Catania, Italy \\ e-mail: 1spezzi@rssd.esa.int \\ 2 European Space Agency (ESTEC), PO Box 299, 2200 AG Noordwijk, The Netherlands \\ Steward Observatory, University of Arizona, 933 North Cherry Avenue, Tucson, AZ 85721, USA \\ 4 Jet Propulsion Laboratory, California Institute of Technology, Pasadena, California 91109-8099, USA \\ European Space Agency (ESAC), PO Box Apdo. de correos 78, 28691 Villanueva de la Cañada, Madrid, Spain \\ ${ }^{6}$ Laboratorio de Astrofísica Espacial y Exoplanetas, Centro de Astrobiologia (LAEFF-CAB, INTA-CSIC), \\ European Space Astronomy Center (ESAC), PO Box 78, 28691 Villanueva de la Cañada, Madrid, Spain \\ Received 15 July 2008 / Accepted 26 February 2009
}

ABSTRACT

\begin{abstract}
Aims. We report the outcome of a deep multiwavelength study of the IC 2391 young open cluster. We attempt to uncover new lowmass and sub-stellar members of the cluster and identify new debris disk objects.

Methods. We observed a $30 \times 30$ square arcmin area in IC 2391 using the wide-field imager at the ESO $2.2 \mathrm{~m}$ telescope. The completeness limits of the photometry at the $3 \sigma$ level are $V=24.7, R_{\mathrm{C}}=23.7$, and $I_{\mathrm{C}}=23.0$, faint enough to reveal sub-stellar members down to $\sim 0.03 M_{\odot}$. Our membership criteria are based on use of our optical data in combination with $J H K_{\mathrm{S}}$ magnitudes from the 2MASS catalog. We also estimate the physical parameters of the selected candidates. Debris disk candidates are identified on the basis of their infrared excess emission using near- and mid-infrared photometry from the Spitzer Space Telescope.

Results. Our optical survey, which has a limiting magnitude at the $3 \sigma$ level 1-2 mag fainter than previous optical surveys conducted in IC 2391, revealed 29 new low-mass member candidates of the cluster. We estimate the contamination to be at least $\sim 50 \%$. We constrain the fraction of sub-stellar objects in the range $8-15 \%$ and discuss possible explanations of the deficit of brown dwarfs in this cluster. We also identify 10 candidates in the cluster showing IR excess emission consistent with the presence of debris disks.
\end{abstract}

Key words. stars: formation - stars: low-mass, brown dwarfs - stars: pre-main sequence - stars: circumstellar matter ISM: individual objects: IC 2391

\section{Introduction}

The general form of the stellar IMF has been observationally established, while the situation remains unclear across the hydrogen-burning limit (Reid 2005). Infrared (IR) survey data (e.g., Bouvier et al. 1998; Lodieu et al. 2005, 2006a,b,c, 2007; Luhman 2004b; Luhman et al. 2006; Moraux et al. 2003; Muench et al. 2003, 2007), have produced reliable statistics for substellar objects. These overall results, in combination with optical photometric and spectroscopic follow-up observations, allow us to address quantitatively such issues as the continuity of the IMF across the stellar/substellar boundary and its invariance or dependency on local conditions. These studies also hold important keys for the clarification of the dominant browndwarf (BD) formation mechanism in star clusters (Bouvier et al. 2005).

Because of its proximity ( $\sim 150 \mathrm{pc}$; Forbes et al. 2001), relatively young age (30-50 Myr; Barrado y Navascués et al. 1999, 2004; Basri et al. 1996; Mermilliod 1981; Stauffer et al. $1998)$, and insignificant extinction along the line of sight $(E(B-$ $V)=0.006$; Patten \& Simon 1996), the IC 2391 young open cluster has been considered to be an ideal location for star-formation studies. Consequently, IC 2391 is one of the most well-studied clusters. In this cluster there are about 180 known members, with accurate proper motion determinations

* Based on observations carried out at the ESO/La Silla, Chile under proposal number 68.D-0541(A).
(Dodd 2004; Monet al. 2003; Platais et al. 2007), among which a dozen are substellar objects. The only determination of the IC 2391 mass spectrum in the very low-mass regime has been presented by Barrado y Navascués et al. (2004). According to these authors, the mass spectrum between $0.5 M_{\odot}$ and the substellar limit $\left(\sim 0.07 M_{\odot}\right)$ follows a power law with $\alpha$-index $\sim 0.96$. Below this limit, there is a sudden drop that the authors attribute to a local drop in the shape of the luminosity-mass relation at spectral types M7-M8 and, partially, to the incompleteness of their spectroscopic follow-up below $I_{C}=18.5$ mag (i.e., $0.05 M_{\odot}$ for cluster members).

However, other young clusters (e.g., $\alpha$ Per and NGC 2547) exhibit a lack of substellar members more or less of the same mass as for IC 2391 (see Barrado y Navascués et al. 2002; Jeffries et al. 2004). This deficit may extend to lower masses or may only be a dip, perhaps caused by an imperfect understanding of the mass-luminosity relationship at temperatures around 2400-2500 K (Dobbie et al. 2002). However, the incompleteness of the surveys conducted in these clusters leaves the question open. In particular, the number of substellar objects discovered so far in IC 2391 is too low to draw any conclusion on a statistically significant basis.

In this paper, we present the results of a deep optical, widefield imaging survey of IC 2391, complemented by IR photometry from the 2MASS catalog (Cutri et al. 2003) and the Spitzer satellite. This survey, which has a $3 \sigma$ photometric limit 1-2 mag fainter than previous optical photometric surveys 
conducted in IC 2391 (i.e., $I_{\mathrm{C}}<21-22$ mag; Barrado y Navascués et al. 2001b; Patten \& Simon 1996; Patten \& Pavlovski 1999), aims to uncover new low-mass and BD member candidates, which should be confirmed with future followup spectroscopy, to provide further insights into the substellar mass spectrum in this cluster. In addition, the age and distance of IC 2391 offer a unique combination in studying the evolution of debris disks around low-mass stars, which is a crucial step toward our understanding of the terrestrial planet formation mechanism (e.g., Currie et al. 2007; Decin et al. 2003; Dominik \& Decin 2003; Habing et al. 2001). The study of circumstellar disks has undergone development following the analyses of data from the Spitzer Space Telescope. These data are ideal for disk investigations because the Spitzer sensitivity and wavelength coverage allow studies of statistically significant samples and probe the inner planet-forming region of disks (see, e.g., Lada et al. 2006). Spitzer observations have shown that debris disks are found around solar-like stars at a wide range of possible distances (1-50 AU) from the central star and temperatures $(10-650 \mathrm{~K})$, with an age-dependent frequency (see Siegler et al. 2007, and reference therein). A pioneer studying of the debris disk population in IC 2391 was conducted by Siegler et al. (2007) using the Multiband Imaging Photometer for Spitzer (MIPS). Using our optical photometry, the MIPS catalog by Siegler et al. (2007), and additional IR photometry from 2MASS and the InfraRed Array Camera (IRAC) on Spitzer, we present here our search for new debris disk candidates in IC 2391. This study aims ultimately to measure the frequency of debris disks around very low-mass stars and assess whether they experience a planetesimal phase in their evolution, as observed for more massive stars (e.g., Chen et al. 2005; Kessler-Silacci et al. 2006; Lada et al. 2006; Riaz et al. 2006; Sicilia-Aguilar et al. 2007; Young et al. 2004).

In Sect. 2, we describe the WFI survey in IC 2391 and the data reduction and catalog extraction procedures. In Sects. 3 and 4, we describe our membership criteria, present the sample of new cluster-member candidates, and estimate the contamination level of this sample. The fraction of substellar objects in IC 2391 is discussed in Sect. 5, while in Sect. 6 we investigate the debris disk population in this cluster. Our conclusions are drawn in Sect. 7.

\section{Observations and data reduction}

\subsection{The WFI observations}

The optical photometric observations were performed in service mode using the Wide Field Imaging (WFI) mosaic camera attached to the ESO $2.2 \mathrm{~m}$ telescope at La Silla (Chile), from November 2001 to March 2002 (Program ID: 68.D-0541(A), PI: Isabella Pagano). WFI is an $8 \mathrm{k} \times 8 \mathrm{k} \mathrm{CCD}$ mosaic, covering a $30 \times 30$ square arcmin field with a pixel scale of 0.238 arcsec/pix. These observations are also intended to investigate the variability of young stars at the bottom of the main sequence caused by their magnetic activity, which will be presented in a future paper (Pagano et al., in preparation). For this reason, our observing strategy consisted of repeated exposures of the same field. To avoid photometric contamination from the bright $A B$ type stars in the core of IC 2391, our WFI pointing was centered on $\mathrm{RA}=08^{\mathrm{h}}: 42^{\mathrm{m}}: 31^{\mathrm{s}}$ and Dec $=-53^{\circ}: 02^{\mathrm{m}}: 58^{\mathrm{s}}$, i.e., about $0.25 \mathrm{deg}$ east of the cluster center (Fig. 1), and covers about $60 \%$ of the sky-area spanned by IC 2391. The region was observed in the $V$, $R$, and $I$ WFI broad-band filters for nine non-consecutive nights, with 1-7 observations per night per filter. The seeing conditions were always superior to 1.2 arcsec. This strategy ensured a good phase coverage for objects with rotational periods between 0.2 and 10 days, i.e., the range expected for very low-mass stars at the age of IC 2391 (30-50 Myr). To cover the gaps between the WFI CCDs, each observation in each filter was divided into three individual exposures (ditherings) and the telescope pointing was shifted by $\sim 1$ arcmin between consecutive exposures. Since we are interested in both identifying the lowest mass cluster members and recovering the early-type ones, we performed two series of observations per night. The total exposure time was $270 \mathrm{~s}$ in the $V$-band and $75 \mathrm{~s}$ in the $R I$ bands for the "long-time" series and $30 \mathrm{~s}$ in the $V$-band, $15 \mathrm{~s}$ in the $R$-band, and $21 \mathrm{~s}$ in the $I$-band for the "short-time" one. In this way, the photometry for earlytype members saturated in the long-time exposures is recovered from the "short-time" ones. A summary of the observations is reported in Table 1.

\subsubsection{Pre-reduction}

The raw images were processed using the IRAF ${ }^{1}$ mscred package and a number of scripts ad hoc developed with both IRAF and $\mathrm{IDL}^{2}$. We followed the standard steps for mosaic CCD data reduction, i.e., overscan, bias and flat-fielding correction. For each night in which observations for our program were performed, bias and twilight-flat frames were combined to obtain the night master bias and flat, respectively, which were then used to correct the science images. The possible residual effects of vignetting and sky concentration caused by the geometrical distortion were reduced by subtracting a background's 2D-polynomial fit from each image, obtained by using the imsurfit routine under IRAF. Fringing strongly affects the WFI observations acquired at near-IR wavelengths. To remove the effect from our I-band images, we subtracted from each $I$-band science frame the fringing pattern frame available as part of the WFI standard calibration plan $^{3}$, scaled by a specific factor to account for the amplitude of the fringes in the individual science frames.

\subsubsection{Astrometry and co-addition of images}

The astrometric calibration of each exposure was performed using the msczero and msccmatch packages under IRAF; the GSC (STScI 2006) and TYCHO (Hog et al. 1998) catalogs were used as reference and an absolute astrometric precision of 0.6 arcsec was achieved, as confirmed by a cross-check with the 2MASS point-source catalog (see Sect. 2.1.4). Individual exposures in each dithering set were then resampled, flux-scaled, and combined into a single mosaic by using the mscimage, mscimatch, and imcombine tasks under IRAF, respectively. The final stacked image is a $8 \mathrm{k} \times 8 \mathrm{k}$ frame, where each pixel value is the median flux of the coadded ditherings normalized to the total exposure time and relative to the airmass and atmospheric transparency of the first frame in the dithering set. The final stacked images were also trimmed to the overlapping area of the relative ditherings, so that only those sources falling in all ditherings were considered in the subsequent analysis.

\footnotetext{
1 IRAF is distributed by NOAO, which is operated by the Association of Universities for Research in Astronomy, Inc., under contract to the National Science Foundation.

2 Interactive Data Language.

3 See: http://www.ls.eso.org/lasilla/sciops/2p2/E2p2M/ WFI/CalPlan/fringing/
} 


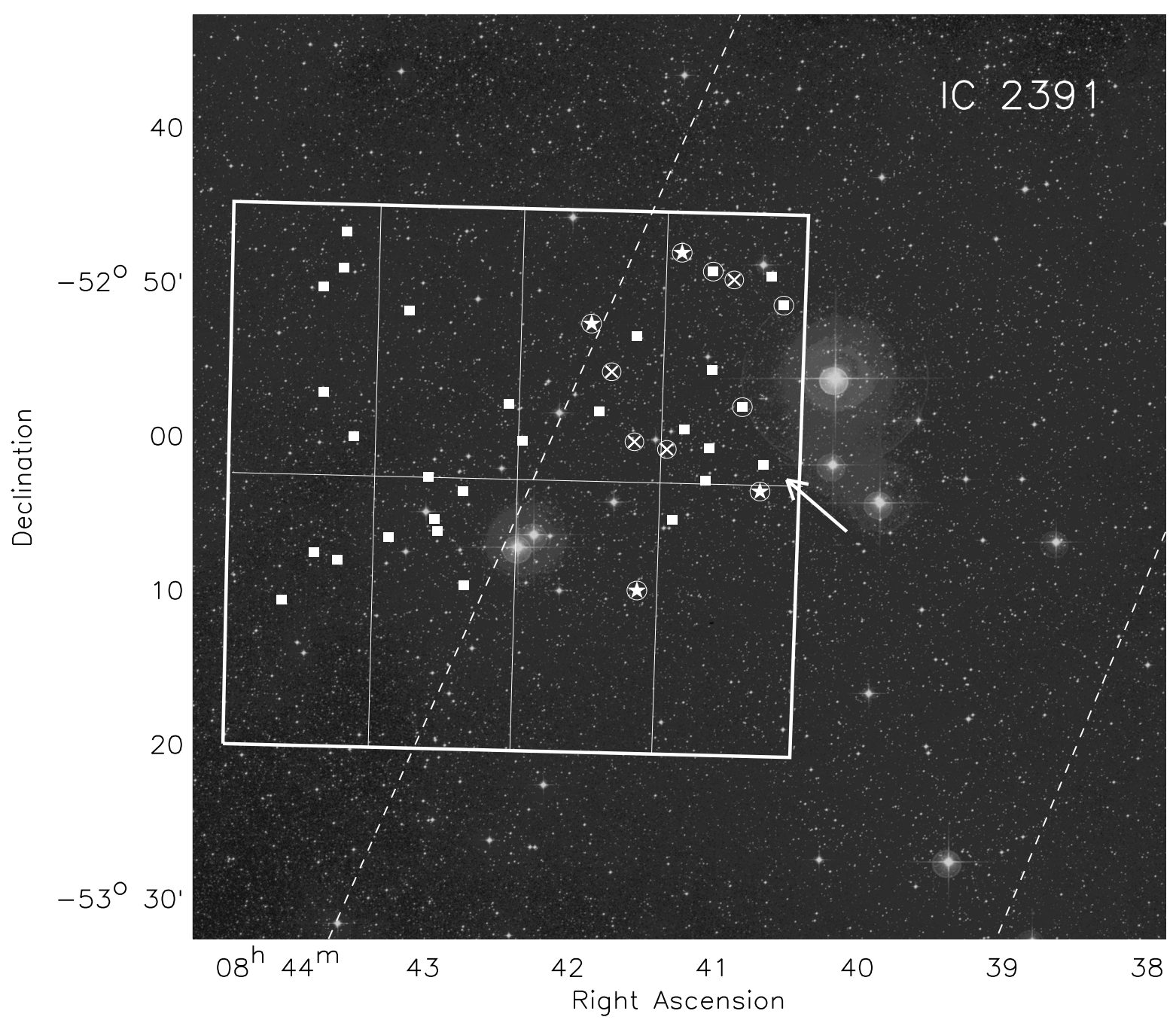

Fig. 1. $R$-band image of IC 2391 from the STScI Digitised Sky Survey. The solid line displays the total sky-area surveyed with the WFI CCD mosaic camera at the ESO $2.2 \mathrm{~m}$ telescope, while the dashed lines trace the area observed with IRAC and MIPS at Spitzer (see Sect. 6). The arrow indicates the cluster center. The filled squares represent the new member candidates identified in this work, while the circled symbols indicate the objects showing IR excess emission (the stars mark the confirmed cluster members, while squares and crosses mark the member candidates and the four sources of dubious nature discussed in Sect. 6.1.1, respectively).

\subsubsection{Photometric calibration}

Instrumental magnitudes were calibrated to the standard Johnson-Cousins system. To achieve this aim, the Landolt's standard star field SA 92 (Landolt 1992) was observed in the VRI filters. By using the IRAF photcal package, we first performed the aperture photometry for the standard stars, obtaining their instrumental magnitudes $\left(v_{0}, r_{0}\right.$, and $\left.i_{0}\right)$ corrected for atmospheric extinction and normalized to the exposure time. Then, the transformation coefficients, namely zero point $(Z P)$ and color term (c), from the WFI photometric system to the Johnson-Cousins standard system were determined by a linear fitting of the following equations:

$V=v_{0}+c_{V} \cdot\left(v_{0}-r_{0}\right)+Z P_{V}$

$R_{\mathrm{C}}=r_{0}+c_{R} \cdot\left(r_{0}-i_{0}\right)+Z P_{R}$

$I_{\mathrm{C}}=i_{0}+c_{I} \cdot\left(r_{0}-i_{0}\right)+Z P_{I}$

where $V, R_{\mathrm{C}}$, and $I_{\mathrm{C}}$ are the standard magnitudes of the Landolt's stars. The mean transformation coefficients determined in our observing run are reported in Table 2 and are consistent with the mean values computed for La Silla ${ }^{4}$.

\subsubsection{The deep catalog extraction}

The sources extraction and photometry from each stacked image in each filter was performed using the SExtractor tool by Bertin $\&$ Arnouts (1996). This data set will be used to study the variability of the IC 2391 low-mass members as will be presented in a future paper (Pagano et al., in preparation). The limiting magnitude achieved at the $3 \sigma$ level using the single stacked images is $R \approx 20$. At the age ( $\sim 50 \mathrm{Myr})$ and distance $(\sim 150 \mathrm{pc})$ to IC 2391 , this limit corresponds to very low-mass stars $\left(M \approx 0.1 M_{\odot}\right)$ close to the hydrogen-burning limits or, perhaps, to massive BDs.

One of the main goals of this work is the identification of new candidate substellar members of IC 2391, which are expected to have $R$ magnitude in the range 18.5-24 depending on their mass and the intervening reddening. Since our data set consists of

\footnotetext{
${ }^{4}$ See http://www.ls.eso.org/lasilla/Telescopes/2p2T/ E2p2M/WFI/zeropoints/
} 
Table 1. Journal of the observations.

\begin{tabular}{|c|c|c|c|c|c|c|c|c|c|}
\hline $\begin{array}{c}\text { Date } \\
(\mathrm{d} / \mathrm{m} / \mathrm{y})\end{array}$ & "WFI Filter & $\overline{\mathrm{MJD}}$ & $\begin{array}{l}T_{\exp } \\
\text { (s) }\end{array}$ & Air Mass & $\begin{array}{c}\text { Date } \\
(\mathrm{d} / \mathrm{m} / \mathrm{y})\end{array}$ & "WFI Filter & $\overline{\mathrm{MJD}}$ & $\begin{array}{l}T_{\exp } \\
\text { (s) }\end{array}$ & Air Mass \\
\hline \multirow[t]{15}{*}{$24 / 11 / 2001$} & $\mathrm{~V} / 89$ & 52237.22128823 & $3 \times 10$ & 1.50 & \multirow[t]{5}{*}{$04 / 03 / 2002$} & $\mathrm{~V} / 89$ & 52337.20706880 & $3 \times 10$ & 1.22 \\
\hline & $\mathrm{Rc} / 162$ & 52237.22634835 & $3 \times 5$ & 1.47 & & $\mathrm{Rc} / 162$ & 52337.21803382 & $3 \times 5$ & 1.25 \\
\hline & Ic/Iwp & 52237.23117713 & $3 \times 7$ & 1.44 & & $\mathrm{Rc} / 162$ & 52337.22950399 & $3 \times 25$ & 1.29 \\
\hline & Ic/Iwp & 52237.23529559 & $3 \times 25$ & 1.42 & & $\mathrm{~V} / 89$ & 52337.23437902 & $3 \times 90$ & 1.31 \\
\hline & $\mathrm{Rc} / 162$ & 52237.24074915 & $3 \times 25$ & 1.39 & & $\mathrm{~V} / 89$ & 52337.24109513 & $3 \times 10$ & 1.34 \\
\hline & $\mathrm{V} / 89$ & 52237.24882797 & $3 \times 90$ & 1.35 & \multirow[t]{5}{*}{$05 / 03 / 2002$} & $\mathrm{~V} / 89$ & 52338.21350087 & $3 \times 10$ & 1.24 \\
\hline & $\mathrm{V} / 89$ & 52237.26303437 & $3 \times 10$ & 1.30 & & $\mathrm{Rc} / 162$ & 52338.21877655 & $3 \times 5$ & 1.26 \\
\hline & $\mathrm{Rc} / 162$ & 52237.26868166 & $3 \times 5$ & 1.28 & & Ic/Iwp & 52338.22356216 & $3 \times 7$ & 1.28 \\
\hline & Ic/Iwp & 52237.27821246 & $3 \times 7$ & 1.24 & & Ic/Iwp & 52338.22763522 & $3 \times 25$ & 1.29 \\
\hline & Ic/Iwp & 52237.28227565 & $3 \times 25$ & 1.23 & & $\mathrm{~V} / 89^{\mathrm{I}}$ & 52338.23909508 & $3 \times 90$ & 1.34 \\
\hline & $\mathrm{Rc} / 162$ & 52237.28773647 & $3 \times 25$ & 1.21 & \multirow[t]{22}{*}{$06 / 03 / 2002$} & $\mathrm{~V} / 89$ & 52339.18778127 & $3 \times 10$ & 1.18 \\
\hline & $\mathrm{V} / 89$ & 52237.29344333 & $3 \times 90$ & 1.20 & & $\mathrm{Rc} / 162$ & 52339.19297910 & $3 \times 5$ & 1.19 \\
\hline & $\mathrm{V} / 89$ & 52237.30066930 & $3 \times 10$ & 1.18 & & Ic/Iwp & 52339.20270214 & $3 \times 7$ & 1.22 \\
\hline & $\mathrm{Rc} / 162$ & 52237.31212718 & $3 \times 5$ & 1.16 & & Ic/Iwp & 52339.20714999 & $3 \times 25$ & 1.23 \\
\hline & Ic/Iwp & 52237.31721340 & $3 \times 7$ & 1.15 & & $\mathrm{Rc} / 162$ & 52339.21242581 & $3 \times 25$ & 1.25 \\
\hline \multirow[t]{12}{*}{$26 / 11 / 2001$} & $\mathrm{~V} / 89$ & 52239.18065529 & $3 \times 10$ & 1.78 & & $\mathrm{~V} / 89$ & 52339.21805674 & $3 \times 90$ & 1.27 \\
\hline & $\mathrm{Rc} / 162$ & 52239.18562202 & $3 \times 5$ & 1.74 & & $\mathrm{~V} / 89$ & 52339.22604426 & $3 \times 10$ & 1.30 \\
\hline & Ic/Iwp & 52239.19029892 & $3 \times 7$ & 1.69 & & $\mathrm{Rc} / 162$ & 52339.23099193 & $3 \times 5$ & 1.32 \\
\hline & Ic/Iwp & 52239.19447743 & $3 \times 25$ & 1.66 & & Ic/Iwp & 52339.23578713 & $3 \times 7$ & 1.34 \\
\hline & $\mathrm{Rc} / 162$ & 52239.19983479 & $3 \times 25$ & 1.62 & & Ic/Iwp & 52339.23999762 & $3 \times 25$ & 1.35 \\
\hline & $\mathrm{V} / 89$ & 52239.20535873 & $3 \times 90$ & 1.57 & & $\mathrm{Rc} / 162$ & 52339.24539390 & $3 \times 25$ & 1.38 \\
\hline & $\mathrm{V} / 89$ & 52239.32447597 & $3 \times 10$ & 1.13 & & $\mathrm{~V} / 89$ & 52339.25120870 & $3 \times 90$ & 1.41 \\
\hline & $\mathrm{Rc} / 162$ & 52239.32957951 & $3 \times 5$ & 1.12 & & $\mathrm{~V} / 89$ & 52339.25913018 & $3 \times 10$ & 1.45 \\
\hline & Ic/Iwp & 52239.33441270 & $3 \times 7$ & 1.12 & & $\mathrm{Rc} / 162$ & 52339.26466470 & $3 \times 5$ & 1.49 \\
\hline & Ic/Iwp & 52239.33845778 & $3 \times 25$ & 1.11 & & Ic/Iwp & 52339.26890995 & $3 \times 7$ & 1.51 \\
\hline & $\mathrm{Rc} / 162$ & 52239.34398065 & $3 \times 25$ & 1.11 & & Ic/Iwp & 52339.27256372 & $3 \times 25$ & 1.53 \\
\hline & $\mathrm{V} / 89$ & 52239.35053232 & $3 \times 90$ & 1.10 & & $\mathrm{Rc} / 162$ & 52339.28297103 & $3 \times 25$ & 1.61 \\
\hline \multirow[t]{11}{*}{$23 / 01 / 2002$} & $V / 89$ & 52297.19774832 & $3 \times 10$ & 1.10 & & $\mathrm{~V} / 89$ & 52339.28856480 & $3 \times 90$ & 1.66 \\
\hline & $\mathrm{Rc} / 162$ & 52297.20344695 & $3 \times 5$ & 1.10 & & $\mathrm{~V} / 89$ & 52339.29614328 & $3 \times 10$ & 1.73 \\
\hline & Ic/Iwp & 52297.20807586 & $3 \times 7$ & 1.09 & & $\mathrm{Rc} / 162$ & 52339.30145210 & $3 \times 5$ & 1.78 \\
\hline & Ic/Iwp & 52297.21229543 & $3 \times 25$ & 1.09 & & Ic/Iwp & 52339.30606411 & $3 \times 7$ & 1.82 \\
\hline & $\mathrm{Rc} / 162$ & 52297.21764270 & $3 \times 25$ & 1.09 & & $\mathrm{Rc} / 162$ & 52339.31534623 & $3 \times 25$ & 1.93 \\
\hline & $\mathrm{V} / 89$ & 52297.22325254 & $3 \times 90$ & 1.09 & \multirow[t]{18}{*}{$24 / 03 / 2002$} & $\mathrm{~V} / 89$ & 52357.07361738 & $3 \times 10$ & 1.10 \\
\hline & $\mathrm{V} / 89$ & 52297.23089695 & $3 \times 10$ & 1.09 & & $\mathrm{Rc} / 162$ & 52357.07891852 & $3 \times 5$ & 1.10 \\
\hline & Ic/Iwp & 52297.24111660 & $3 \times 7$ & 1.10 & & Ic/Iwp & 52357.08412627 & $3 \times 7$ & 1.10 \\
\hline & Ic/Iwp & 52297.24521964 & $3 \times 25$ & 1.10 & & Ic/Iwp & 52357.08840132 & $3 \times 25$ & 1.10 \\
\hline & $\mathrm{Rc} / 162$ & 52297.25143005 & $3 \times 25$ & 1.10 & & $\mathrm{Rc} / 162$ & 52357.09377871 & $3 \times 25$ & 1.11 \\
\hline & $\mathrm{V} / 89$ & 52297.25701984 & $3 \times 90$ & 1.11 & & $\mathrm{~V} / 89$ & 52357.09933803 & $3 \times 90$ & 1.12 \\
\hline \multirow[t]{12}{*}{$11 / 02 / 2002$} & $\mathrm{~V} / 89$ & 52316.14784279 & $3 \times 10$ & 1.10 & & $\mathrm{~V} / 89$ & 52357.10984608 & $3 \times 10$ & 1.13 \\
\hline & $\mathrm{Rc} / 162$ & 52316.15586182 & $3 \times 5$ & 1.09 & & $\mathrm{Rc} / 162$ & 52357.12092981 & $3 \times 5$ & 1.15 \\
\hline & Ic/Iwp & 52316.16066498 & $3 \times 7$ & 1.09 & & Ic/Iwp & 52357.12572673 & $3 \times 7$ & 1.16 \\
\hline & Ic/Iwp & 52316.16510996 & $3 \times 25$ & 1.09 & & Ic/Iwp & 52357.13007714 & $3 \times 25$ & 1.16 \\
\hline & $\mathrm{Rc} / 162$ & 52316.17086954 & $3 \times 25$ & 1.09 & & $\mathrm{Rc} / 162$ & 52357.13532178 & $3 \times 25$ & 1.17 \\
\hline & $\mathrm{V} / 89$ & 52316.17698296 & $3 \times 90$ & 1.09 & & $\mathrm{~V} / 89$ & 52357.16004606 & $3 \times 10$ & 1.24 \\
\hline & $\mathrm{V} / 89$ & 52316.18480783 & $3 \times 10$ & 1.10 & & $\mathrm{Rc} / 162$ & 52357.16513120 & $3 \times 5$ & 1.26 \\
\hline & $\mathrm{Rc} / 162$ & 52316.19440469 & $3 \times 5$ & 1.10 & & Ic/Iwp & 52357.16995618 & $3 \times 7$ & 1.27 \\
\hline & Ic/Iwp & 52316.19919322 & $3 \times 7$ & 1.10 & & Ic/Iwp & 52357.17390633 & $3 \times 25$ & 1.28 \\
\hline & Ic/Iwp & 52316.20328731 & $3 \times 25$ & 1.11 & & $\mathrm{Rc} / 162$ & 52357.18004707 & $3 \times 25$ & 1.31 \\
\hline & $\mathrm{Rc} / 162$ & 52316.20891675 & $3 \times 25$ & 1.11 & & $\mathrm{~V} / 89$ & 52357.18570452 & $3 \times 90$ & 1.33 \\
\hline & $\mathrm{V} / 89$ & 52316.21545996 & $3 \times 90$ & 1.12 & & $\mathrm{~V} / 89$ & 52357.99572072 & $3 \times 10$ & 1.13 \\
\hline \multirow[t]{5}{*}{$24 / 02 / 2002$} & $\mathrm{~V} / 89$ & 52329.19058819 & $3 \times 10$ & 1.14 & \multirow[t]{5}{*}{$25 / 03 / 2002$} & $\mathrm{Rc} / 162$ & 52358.00131936 & $3 \times 5$ & 1.13 \\
\hline & $\mathrm{Rc} / 162$ & 52329.19609543 & $3 \times 5$ & 1.14 & & Ic/Iwp & 52358.00615478 & $3 \times 7$ & 1.12 \\
\hline & Ic/Iwp & 52329.20090155 & $3 \times 7$ & 1.15 & & $\mathrm{Rc} / 162$ & 52358.01631727 & $3 \times 25$ & 1.11 \\
\hline & $\mathrm{Rc} / 162$ & 52329.21181380 & $3 \times 25$ & 1.17 & & $\mathrm{~V} / 89$ & 52358.02278763 & $3 \times 90$ & 1.10 \\
\hline & $\mathrm{V} / 89$ & 52329.21777151 & $3 \times 90$ & 1.19 & & & & & \\
\hline
\end{tabular}

repeated observations of the same field, we further combined the stacked images with the same exposure time and obtained, for each filter, a photometrically deeper frame with improved signalto-noise ratio, suitable for our purposes (Fig. 2). The combination was performed using the imcombine task under IRAF in the same configuration as explained in Sect. 2.1.2, and the stacked images from our "long-time" series (see Sect. 2.1). Seventeen images were combined in the $V$ filter, 18 in the $R$ filter, and 15 in the $I$ filter, for a total exposure time of $76.5 \mathrm{~min}, 22.5 \mathrm{~min}$, and $18.75 \mathrm{~min}$, respectively. PSF-fitting photometry was performed on these deeper frames by using the IRAF daophot package (Stetson 1987); this method provides a more accurate deblending of sources than aperture photometry and hence, the detection of the faintest neighbors to bright stars. Since we are interested 
Table 2. Mean photometric calibration coefficients for our WFI observing run.

\begin{tabular}{cccc}
\hline \hline Filter & $A E^{\dagger}$ & $Z P$ & $c$ \\
\hline$V$ & 0.130 & $24.195 \pm 0.035$ & $0.071 \pm 0.037$ \\
$R$ & 0.096 & $24.509 \pm 0.027$ & $0.078 \pm 0.031$ \\
$I$ & 0.082 & $23.502 \pm 0.034$ & $0.325 \pm 0.044$ \\
\hline
\end{tabular}

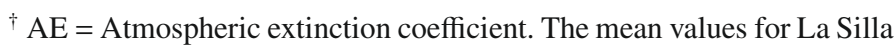
have been adopted.

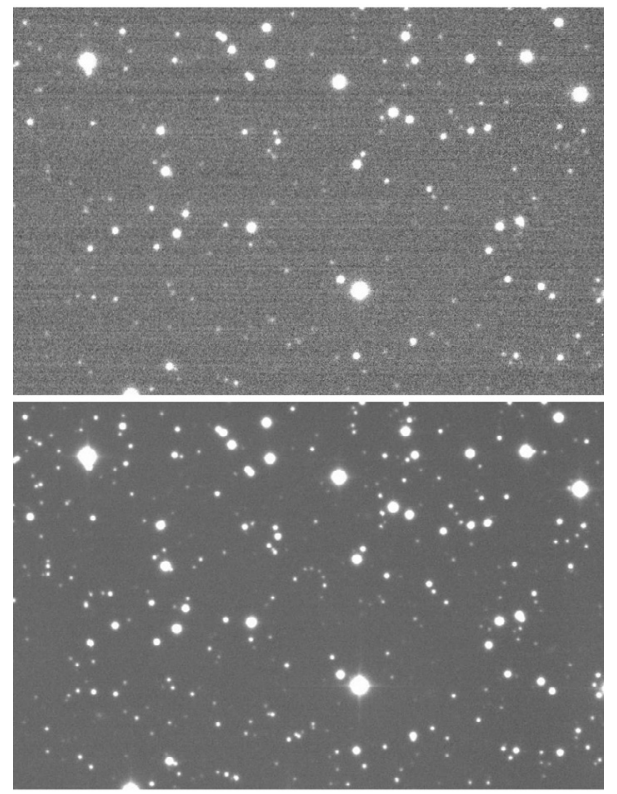

Fig. 2. Section of a single stacked image in the $R$-band (upper panel) and the same region as it appears in the deeper frame obtained by combining 18 stacked images (lower panel).

in very low-luminosity objects, we defined a very low detection threshold $(\sigma=3)$ and selected point-like objects from extended sources (e.g., galaxies), saturated objects, and other spurious detections by using the daophot morphological parameters. In Fig. 3, the internal photometric errors of all the detected pointlike sources are plotted against magnitude for all the available filters; the relative exponential fits are overplotted. Table 3 reports the saturation limits and the limiting magnitudes achieved at the $3 \sigma$ level from these deeper frames. These limits are sufficient for the detection of young stars and BDs with masses of between $\sim 1 M_{\odot}$ and $\sim 0.03 M_{\odot}$ at the age and distance of IC 2391.

Our optical catalog contains 60596 point-like sources with VRI magnitudes. A merged catalog with position and photometry in each optical filter, as well as in the near-IR ( $J H K_{\mathrm{S}}$ magnitudes from 2MASS; Cutri et al. 2003), was finally produced and used for the selection of cluster members (Sect. 3). A matching radius of $1^{\prime \prime}$ was used to merge the WFI and the 2MASS catalogs; this value was set by taking the astrometric accuracy of both catalogs into account.

\subsubsection{Completeness}

The completeness of our "deep" catalog was derived empirically. We used the IRAF addstar package to add test stars into the frames and then the same PSF-fitting procedure as for true objects (Sect. 2.1.4) to determine the fraction of these artificial stars recoverable as a function of magnitude. This fraction provides a measure of the completeness. We inserted 500 artificial
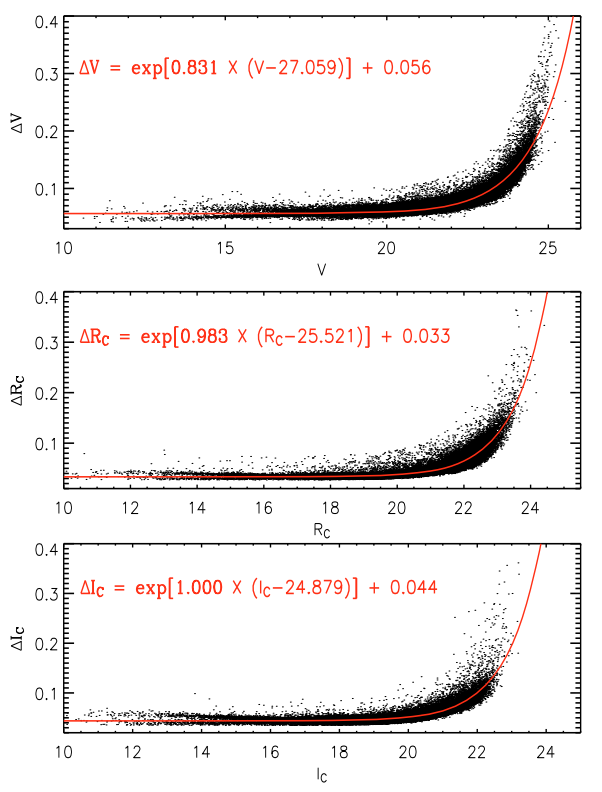

Fig. 3. Photometric errors versus magnitudes and relative exponential fits of all the point-like sources detected in the $30 \times 30$ square arcmin surveyed area in IC 2391. Magnitudes and errors are from the deep frames obtained as discussed in Sect. 2.1.4.

Table 3. Saturation limits and limiting magnitudes at $3 \sigma$ level (see Sect. 2.1.4). In the fourth column, the corresponding limiting mass for cluster members (i.e. assuming an age between 30 and $50 \mathrm{Myr}$ and a distance of $150 \mathrm{pc}$ ) derived from the evolutionary models by Baraffe et al. (1998) and Chabrier et al. (2000) is reported; in the last column the magnitude corresponding to the Hydrogen burning limit $\left(\sim 0.07 M_{\odot}\right)$ for IC 2391 members is also given for comparison.

\begin{tabular}{ccccc}
\hline \hline Filter & Mag Sat. & Mag 3 $\sigma$ & $\begin{array}{c}\text { Mass } 3 \sigma \\
\left(M_{\odot}\right)\end{array}$ & PHB $^{\dagger}$ \\
\hline$V$ & 10.5 & 24.7 & 0.03 & 20.0 \\
$R_{\mathrm{C}}$ & 10.0 & 23.7 & 0.03 & 18.5 \\
$I_{\mathrm{C}}$ & 9.5 & 23.0 & 0.02 & 17.0 \\
\hline
\end{tabular}

${ }^{\dagger}$ PHB $=$ Photometric Hydrogen Burning limit for IC 2391 members.

sources into each of the three deep frames in the VRI filters; this number is statistically significant for our purposes and, at the same time, does not alter the crowding in the images. The profile for the artificial sources was generated by using the same PSF model as used for the source extraction; the positions of the artificial objects were randomly distributed over the entire area of the mosaic, and their magnitudes range uniformly between the detection and the saturation limits in each band (Table 3). Figure 4 shows the fraction of recovered artificial objects, i.e., the completeness (C), as a function of magnitude for each filter. We estimate a $100 \%$ completeness level down to $V \approx 20$, $R \approx 19.5$, and $I \approx 18.5$; however, objects down to our detection limits at $3 \sigma$ level (Table 3 ) are recovered with a completeness level of higher than $\sim 80 \%$. Using the theoretical isochrones and evolutionary tracks of Baraffe et al. (1998) and Chabrier et al. (2000) for the WFI-Cousins system (see Sect. 3.1 by Spezzi et al. 2007) and considering the age and distance of IC 2391, we estimate that the $100 \%$ and $80 \%$ completeness levels correspond to objects with $M \approx 0.05 M_{\odot}$ and $M \approx 0.03 M_{\odot}$, respectively. Interstellar extinction effects are expected to be negligible (see Sect. 1). 


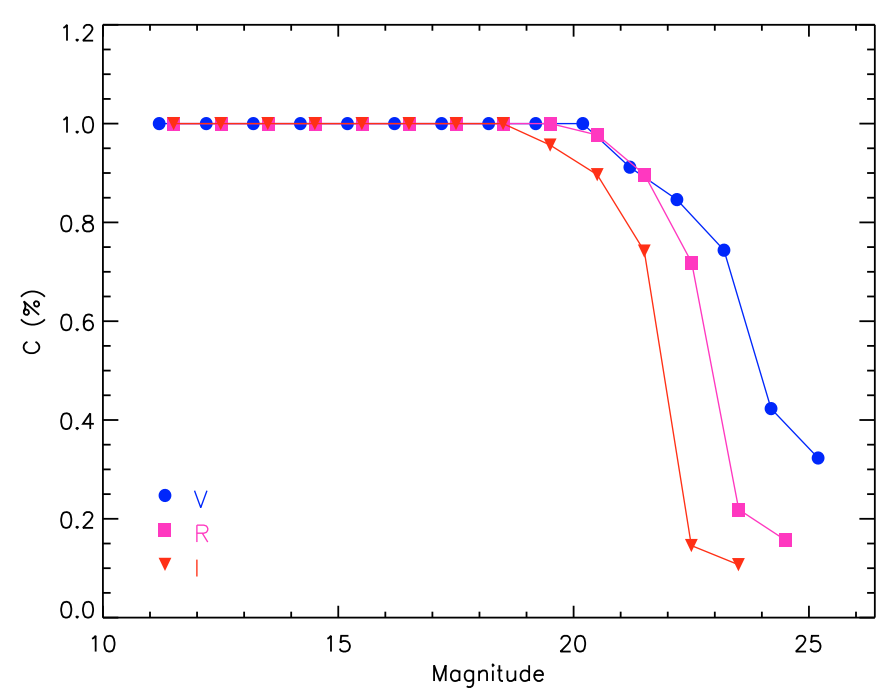

Fig. 4. Completeness (C) plot for extraction of artificial stars from our "deep" mosaics (see Sect. 2.1.4) for the three optical bands used in this work.

\subsection{Spitzer observations and data reduction}

IRAC (Fazio et al. 2004) observations of IC 2391 were obtained on 30 December 2006. The $12 \mathrm{~s}$ high-dynamic-range mode was used to obtain two frames in each position, one with $0.4 \mathrm{~s}$ exposure time and one with $10.4 \mathrm{~s}$. The observation of each field was repeated three times with a small offset, providing $31.2 \mathrm{~s}$ integration time for each position. The frames were processed using the SSC IRAC Pipeline v15.05, and mosaics were created from the basic calibrated data (BCD) frames using a customized IDL program (see Gutermuth et al. 2008, for details). Aperture photometry for these images was carried out using PhotVis version 1.10, which is an IDL-GUI based photometry visualization tool (see Gutermuth et al. 2004 for further details about PhotVis). The radii of the source aperture, and the inner and outer boundaries of the sky annulus were 2.4, 2.4, and 7.2 arcsec, respectively. The calibration used large-aperture measurements of standard stars. The zero-point magnitudes of the calibration were $19.6642,18.9276,16.8468$, and 17.3909 for channel 1,2, 3 , and 4, respectively. Aperture corrections of $0.21,0.23,0.35$, and 0.5 mag were applied to channels $1,2,3$, and 4 to account for the differences between the aperture sizes used for the standard stars and those used for the IC 2391 photometry. The sensitivity of the IRAC observations is sufficient for the detection of objects with $K_{\mathrm{S}} \lesssim 14.5$, which corresponds to the hydrogen-burning limit for cluster members. MIPS observations in IC 2391 and the relative data reduction and calibration were described by Siegler et al. (2007). The standard fluxes at each Spitzer passband were derived from the observed magnitudes using the absolute flux calibration constants by Reach et al. (2005) and Engelbracht et al. (2007) for IRAC and MIPS/24 $\mu \mathrm{m}$ bands, respectively.

\section{Selection of new cluster members}

The extraction of member candidates in open clusters generally involves selecting objects whose position in color-magnitude diagrams (CMDs) is located above the ZAMS shifted to the distance of the cluster (see, e.g., Barrado y Navascués et al. 2001a; Leggett 1992; Lodieu 2005). The selection of IC 2391 member candidates was carried out using a similar criterion, refined by exploiting estimates of the cluster proper motion and the photometry of previously known cluster members available from the literature. Our selection procedure includes the three steps that are now described.

\subsection{Determination of stellar parameters}

We first assumed that all the point-like objects detected in our images above $3 \sigma$ level are all at the distance of IC 2391 and simultaneously estimated their visual extinction $\left(A_{V}\right)$, effective temperature $\left(T_{\text {eff }}\right)$, and stellar radius $\left(R_{\star}\right)$ by fitting a grid of stellar photosphere models to their observed spectral energy distribution (SED). The observed SEDs were derived by merging the VRI WFI-Cousins photometry with the 2MASS $J H K_{\mathrm{S}}$ photometry; the standard fluxes at each passband were derived from the observed magnitudes using the absolute flux calibration constants by Johnson (1965) and Cousins (1976) for the optical bands and Cutri et al. (2003) for the 2MASS bands, respectively. The grid of reference SEDs was constructed by using the NextGen and Stardusty model spectra by Hauschildt et al. (1999) and Allard et al. (2000), and the WFI and 2MASS filter transmission curves, as prescribed by Spezzi et al. (2007). Our grid spans the $T_{\text {eff }}$ range $1700-10000 \mathrm{~K}$ and assumes a surface gravity of $\log g=4.5$, as expected for low-mass objects at the age of IC 2391. The reference SEDs were scaled to the distance of IC 2391 (i.e., 150 pc) and fitted to the observed SED of each object following the prescription by Spezzi et al. (2007) and adopting the extinction law by Cardelli et al. $(1989)^{5}$. The $A_{V}, T_{\text {eff }}$, and $R_{\star}$ values of the reference SED minimizing the $\chi^{2}$ of the fit correspond to the closest approximation to the true stellar parameters. The stellar luminosity $\left(L_{\star}\right)$ is deriving to be $L_{\star}=4 \pi \sigma R_{\star 2} T_{\text {eff }}^{4}$, where $\sigma$ is the Stefan-Boltzmann constant. For more details about the SED fitting procedure, we refer the reader to Spezzi et al. (2007). The goodness of our fits can be appreciated from the examples shown in Fig. 8.

As demonstrated by Spezzi et al. (2007), this parametrization method turns out to be accurate to within about $250 \mathrm{~K}$ in $T_{\text {eff }}$ and $1.5 \mathrm{mag}$ in $A_{V}$ for objects with spectral type later than K5 (i.e. $T_{\text {eff }} \lesssim 4500 \mathrm{~K}$ ). These uncertainties translate into an average uncertainty of 0.15 dex in the logarithm of luminosity. This accuracy in the derived physical parameters is sufficient for candidate selection purposes. On the basis of the saturation limits of our survey (Table 3), we expect to recover only the low-mass cluster members $\left(R_{\mathrm{C}} \gtrsim 10 \mathrm{mag}\right.$, i.e. $M \lessgtr 1 M_{\odot}$ at the age and distance of IC 2391), for which the parametrization method by Spezzi et al. (2007) can be confidently applied. Note also that possible IR excesses at 2MASS wavelengths due to the presence of hot circumstellar dust does not appear to be a major issue in our fits. Indeed, the age of IC 2391 is consistent with both theoretical and observational timescales of disk dissipation and terrestrial planet formation (Chambers 2001; Kleine et al. 2002; Siegler et al. 2007); thus, following the classification by Lada (1987) as extended by Greene et al. (1994), the cluster population is expected to consist mainly of class III objects with no prominent IR excess at nearIR wavelengths. Moreover, the same parametrization procedure was applied to 40 confirmed members of IC 2391 whose spectral type (later than K5) and optical/near-IR photometry were recovered from the literature (Barrado y Navascués et al. 1999, 2001b, 2004; Cutri et al. 2003; Patten \& Simon 1996; Patten \& Pavlovski 1999; Rolleston et al. 1997; Simon \& Patten 1998).

\footnotetext{
5 We also checked that using other extinction laws, such as those by Savage \& Mathis (1979) and Weingartner \& Draine (2001), does not affect significantly our results. We adopt the Cardelli et al. (1989) extinction law because its analytic form is more functional for our purposes.
} 


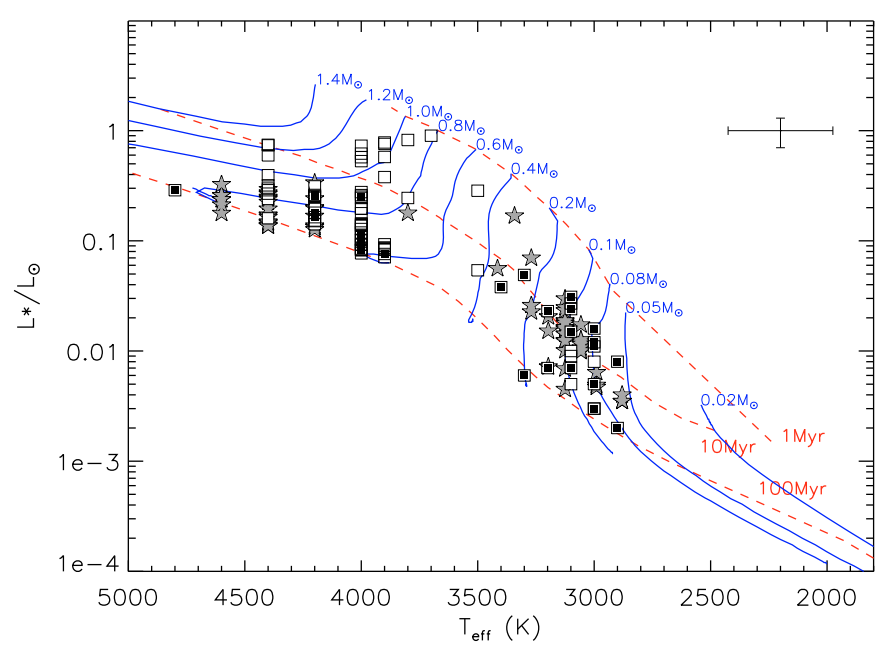

Fig. 5. HR diagram for 40 low-mass confirmed members of IC 2391 (stars), the sample of optical sources whose isochronal age is $<100 \mathrm{Myr}$ (open squares; see Sect. 3.2) and the final sample of 29 member candidates selected in this work (filled squares). The continuous and dashed lines are the evolutionary tracks and isochrone by Baraffe et al. (1998) and Chabrier et al. (2000). The average error bars on effective temperature and luminosity are shown in the top-right.

Our fitting procedure yields $T_{\text {eff }}$ and $A_{V}$ values for these objects that are consistent with those derived from spectroscopy within $\sim 250 \mathrm{~K}$ and $\sim 0.7 \mathrm{mag}$, respectively. This further supports the reliability of our parametrization method.

\subsection{Selection of cluster member candidate from the HR diagram}

Having a reliable estimate of $T_{\text {eff }}$ and $L_{\star}$ for all the sources in our catalog with $T_{\text {eff }} \lesssim 4500 \mathrm{~K}$ (i.e. 2395 sources), we constructed the Hertzsprung-Russell (HR) diagram for these sources and for the 40 confirmed members of IC 2391 mentioned above (Sect. 3.1). Mass and age of these objects can now be determined by comparison of their location on the HR diagram with a suitable set of evolutionary tracks, which in our case are those of Baraffe et al. (1998) and Chabrier et al. (2000). We found that the isochronal age of the 40 low-mass cluster members ranges between 10 and $80 \mathrm{Myr}$, and has a mean value of 20$30 \mathrm{Myr}$ (Fig. 5); this isochronal age is in fair agreement with the cluster age estimated on the basis of the lithium-depletion technique and the main-sequence isochrone fitting (30-50 Myr; Barrado y Navascués et al. 1999, 2004; Basri et al. 1996; Mermilliod 1981; Stauffer et al. 1998). However, we observe an age spread of between a few Myr and 100 Myr (see also Table 5), which is wider than reported in the literature. This wider age spread mainly arises from our indetermination on effective temperature and luminosity (Sect. 3.1); indeed, stellar parameters inferred from photometry alone are far more inaccurate than those derived from spectroscopy, although still useful for candidate selection purposes.

New low-mass cluster members are expected to be found in the same locus of the HR diagram. Thus, we performed a firstlevel selection of member candidates by selecting objects whose isochronal age is $<100 \mathrm{Myr}$; this selection yielded 90 candidates. We also identified a large number of sources in our catalog for which we could not assign an age because their location on the
HR diagram is inconsistent with the pre-main sequence (PMS) locus when the distance of IC 2391 is assumed; these sources are field objects unrelated to the cluster.

The advantage of using this approach, compared to frequently used membership criteria based on CMDs, is in the simultaneous use of the photometric information from the $V$ to the $K_{\mathrm{S}}$ bands; this wavelength range contains the peak of the photospheric emission of cool, low-mass objects, and enables a highly reliable determination of the stellar parameters (Comerón et al., $\mathrm{A} \& \mathrm{~A}$, in press).

\subsection{Refining the sample using proper motion measurements}

Figure 6 show the $R_{\mathrm{C}}$ vs. $\left(V-R_{\mathrm{C}}\right)$ and $I_{\mathrm{C}}$ vs. $(R-I)_{\mathrm{C}} \mathrm{CMDs}$ for all the point-like optical sources detected in IC 2391 above $3 \sigma$ level and the 90 candidates selected from the inspection of the HR diagram. The lines in these plots are the 1 and 50 Myr theoretical NextGen isochrones by Baraffe et al. (1998) for effective temperatures higher than $2500 \mathrm{~K}$ and the DUSTY isochrones by Chabrier et al. (2000) for lower temperatures; these isochrones were transformed into the WFI-Cousins system, as described by Spezzi et al. (2007), and scaled to the IC 2391 distance of $150 \mathrm{pc}$. No correction was made for the mean reddening towards IC 2391, which is estimated to be close to zero $(E(B-V)=0.006$; Patten \& Simon 1996). Both diagrams show a wide sequence of candidates with $12 \lesssim R_{\mathrm{C}} \lesssim 15 \mathrm{mag}$ and $11 \lesssim I_{\mathrm{C}} \lesssim 14 \mathrm{mag}$, which is probably caused by the merging of the cluster and field star sequences. Since the Galactic latitude of IC 2391 is about $-7^{\circ}$, it is indeed inevitable that the sample of photometrically selected candidates is contaminated by field stars.

To remove stellar contaminants, the proper motions of cluster member candidates are generally computed and compared to those of field objects (see, e.g., Casewell et al. 2007). We applied this proper motion analysis to the candidates in our sample using two proper motion catalogs: the all-sky Naval Observatory Merged Astrometric Dataset (NOMAD Zacharias et al. 2005), reporting proper motions for stars down to $R_{\mathrm{C}} \approx 16$ mag with an average accuracy of 6 mas/yr, and the catalog presented by Platais et al. (2007), who obtained proper motions for 6991 stars down to $V \approx 13-16$ over a $\sim 9 \mathrm{deg}^{2}$ area around IC 2391 with a precision of about 1.7 mas/yr. These two catalogs reported consistent proper motions for the confirmed cluster members, although the NOMAD catalog is photometrically deeper and hence, we found a much higher number of matches with our candidate list than with the Platais et al. (2007) catalog. Thus, we used the proper motions from NOMAD, where 70 candidates out of 90 in our original list (Sect. 3.2) have proper motion estimates. Because of the low precision, we were unable to calculate the probability of membership for the standard method described in Moraux et al. (2003). Consequently, we only selected objects to be proper motion members if their proper motions were within $3 \sigma$ of the cluster mean proper motion $(p m(\mathrm{RA}) \approx-24 \mathrm{mas} / \mathrm{yr}$ and $p m(\mathrm{DEC}) \approx+24$ mas/yr; Dodd 2004; Kharchenko 2001). This criterion identified only 9 proper motion member candidates out of 70 . Adding these objects to the 20 fainter candidates whose proper motion are not available, we obtained a sample of 29 new member candidates of IC 2391. Of course, we still expected a certain level of contamination, especially among the candidates without proper motion estimates; this issue is discussed in Sect. 4. 
Table 4. Optical and near-IR photometry for the IC 2391 members and candidates of IC 2391 discussed in this work. The objects with identification number (ID) from 1 to 29 are the new candidates selected in this work (Sect. 3); three of these candidates show IR excess emission at $24 \mu \mathrm{m}$ and are marked by an asterisk. The objects with ID from 30 to 37 are the additional 8 cluster members and candidates showing IR excess emission discussed in Sect. 6.

\begin{tabular}{|c|c|c|c|c|c|c|c|c|c|c|c|c|}
\hline ID & Designation & 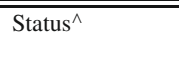 & Ref. ${ }^{\bullet}$ & $\begin{array}{c}\mathrm{RA}_{J 2000}^{\dagger} \\
\text { (hh:mm:ss) }\end{array}$ & $\begin{array}{c}\operatorname{Dec}_{J 2000}^{\dagger} \\
\text { (dd:mm:ss) }\end{array}$ & $\overline{B^{\diamond}}$ & $\overline{V^{\dagger}}$ & $\overline{\overline{R_{\mathrm{c}}^{\dagger}}}$ & $\overline{I_{\mathrm{c}}^{\dagger}}$ & $\overline{J^{\natural}}$ & $\overline{H^{\ddagger}}$ & $\overline{K_{\mathrm{S}}^{\natural}}$ \\
\hline 1 & WFI J08403964-5250429* & HR-C & & $08: 40: 39.643$ & $-52: 50: 42.86$ & - & $21.24 \pm 0.09$ & $19.82 \pm 0.05$ & $17.83 \pm 0.07$ & $15.54 \pm 0.05$ & $14.86 \pm 0.08$ & $14.52 \pm 0.09$ \\
\hline 2 & WFI J08404512-5248521 & HR-C, PM-C & & 08:40:45.118 & $-52: 48: 52.09$ & 17.10 & $16.60 \pm 0.07$ & $15.39 \pm 0.03$ & $13.97 \pm 0.04$ & $12.23 \pm 0.02$ & $11.65 \pm 0.02$ & $11.42 \pm 0.02$ \\
\hline 3 & WFI J08404578-5301052 & HR-C & & $08: 40: 45.780$ & $-53: 01: 05.23$ & - & $14.50 \pm 0.07$ & $13.36 \pm 0.03$ & $12.63 \pm 0.04$ & $11.24 \pm 0.03$ & $10.43 \pm 0.04$ & $10.19 \pm 0.05$ \\
\hline 4 & WFI J08405518-5257227 & HR-C & & 08:40:55.176 & $-52: 57: 22.68$ & 15.52 & $14.59 \pm 0.07$ & $13.39 \pm 0.03$ & $12.58 \pm 0.04$ & $10.94 \pm 0.03$ & $10.10 \pm 0.03$ & $9.84 \pm 0.02$ \\
\hline 5 & WFI J08410806-5300053 ${ }^{\mathrm{OD}}$ & HR-C, X & a & 08:41:08.057 & $-53: 00: 05.26$ & - & $18.02 \pm 0.08$ & $16.82 \pm 0.06$ & $15.27 \pm 0.06$ & $13.28 \pm 0.03$ & $12.67 \pm 0.06$ & $12.45 \pm 0.05$ \\
\hline 6 & WFI J08410814-5255027 & HR-C, PM-C & & 08:41:08.141 & $-52: 55: 02.71$ & 14.30 & $14.69 \pm 0.07$ & $13.45 \pm 0.03$ & $12.64 \pm 0.04$ & $10.98 \pm 0.04$ & $10.08 \pm 0.04$ & $9.81 \pm 0.03$ \\
\hline 7 & WFI J08410886-5248387* & HR-C, X & & 08:41:08.856 & $-52: 48: 38.74$ & 18.77 & $18.74 \pm 0.07$ & $17.46 \pm 0.04$ & $15.66 \pm 0.06$ & $13.52 \pm 0.03$ & $12.90 \pm 0.02$ & $12.65 \pm 0.03$ \\
\hline 8 & WFI J08410937-5302136 & HR-C, X & & 08:41:09.374 & $-53: 02: 13.60$ & 17.51 & $17.58 \pm 0.07$ & $16.38 \pm 0.04$ & $14.74 \pm 0.05$ & $12.73 \pm 0.03$ & $12.11 \pm 0.03$ & $11.91 \pm 0.03$ \\
\hline 9 & WFI J08411865-5258549OD & HR-C, X & $\mathrm{a}$ & $08: 41: 18.650$ & $-52: 58: 54.88$ & 19.61 & $18.83 \pm 0.07$ & $17.64 \pm 0.03$ & $16.12 \pm 0.05$ & $14.14 \pm 0.03$ & $13.58 \pm 0.03$ & $13.23 \pm 0.04$ \\
\hline 10 & WFI J08412229-5304471 & HR-C, X & & $08: 41: 22.291$ & $-53: 04: 47.14$ & - 17.77 & $17.16 \pm 0.07$ & $15.98 \pm 0.03$ & $14.43 \pm 0.05$ & $12.47 \pm 0.02$ & $11.85 \pm 0.02$ & $11.58 \pm 0.02$ \\
\hline 11 & WFI J08413933-5252565 & HR-C & & $08: 41: 39.326$ & $-52: 52: 56.53$ & 15.76 & $14.93 \pm 0.07$ & $13.81 \pm 0.03$ & $13.09 \pm 0.04$ & $11.60 \pm 0.02$ & $10.85 \pm 0.03$ & $10.65 \pm 0.02$ \\
\hline 12 & WFI J08415360-5257558 & HR-C, X & & 08:41:53.602 & $-52: 57: 55.80$ & 18.12 & $17.44 \pm 0.07$ & $16.24 \pm 0.03$ & $14.72 \pm 0.05$ & $12.79 \pm 0.03$ & $12.17 \pm 0.02$ & $11.93 \pm 0.03$ \\
\hline 13 & WFI J08422460-5259575 & HR-C, PM-C & & $08: 42: 24.605$ & $-52: 59: 57.48$ & 13.71 & $12.71 \pm 0.05$ & $11.91 \pm 0.04$ & $11.48 \pm 0.05$ & $10.54 \pm 0.03$ & $10.05 \pm 0.03$ & $9.92 \pm 0.02$ \\
\hline 15 & WFI J08424731-5309235 & HR-C, X & & $08: 42: 47.314$ & $-53: 09: 23.54$ & 17.80 & $20.36 \pm 0.08$ & $18.91 \pm 0.05$ & $16.79 \pm 0.07$ & $14.20 \pm 0.05$ & $13.64 \pm 0.05$ & $13.24 \pm 0.04$ \\
\hline 16 & WFI J08424850-5303187 & HR-C, X & & $08: 42: 48.502$ & $-53: 03: 18.72$ & 19.07 & $18.55 \pm 0.07$ & $17.26 \pm 0.04$ & $15.63 \pm 0.05$ & $13.59 \pm 0.02$ & $12.99 \pm 0.02$ & $12.68 \pm 0.03$ \\
\hline 17 & WFI J08425873-5305546 & HR-C, PM-C, X & & $08: 42: 58.733$ & $-53: 05: 54.64$ & 17.56 & $16.55 \pm 0.07$ & $15.33 \pm 0.03$ & $13.87 \pm 0.04$ & $12.01 \pm 0.02$ & $11.38 \pm 0.02$ & $11.13 \pm 0.02$ \\
\hline 18 & WFI J08425988-5305074 & HR-C & & $08: 42: 59.880$ & $-53: 05: 07.44$ & - & $20.65 \pm 0.08$ & $19.19 \pm 0.05$ & $17.17 \pm 0.07$ & $14.77 \pm 0.04$ & $14.16 \pm 0.04$ & $13.81 \pm 0.06$ \\
\hline 19 & WFI J08430294-5302259 & HR-C & & 08:43:02.938 & $-53: 02: 25.91$ & 15.07 & $13.97 \pm 0.07$ & $12.87 \pm 0.03$ & $12.10 \pm 0.04$ & $10.60 \pm 0.02$ & $9.79 \pm 0.02$ & $9.59 \pm 0.02$ \\
\hline 20 & WFI J08431228-5251411 & HR-C & & $08: 43: 12.283$ & $-52: 51: 41.11$ & - & $22.85 \pm 0.10$ & $21.32 \pm 0.07$ & $18.92 \pm 0.09$ & $16.18 \pm 0.09$ & $15.53 \pm 0.13$ & $15.45^{U L}$ \\
\hline 21 & WFI J08431860-5306241 & HR-C,PM-C & & 08:43:18.602 & $-53: 06: 24.12$ & 13.97 & $13.41 \pm 0.06$ & $12.43 \pm 0.03$ & $11.80 \pm 0.04$ & $10.52 \pm 0.02$ & $9.83 \pm 0.02$ & $9.67 \pm 0.02$ \\
\hline 22 & WFI J08433366-5259549 & HR-C & & $08: 43: 33.658$ & $-52: 59: 54.89$ & - & $22.71 \pm 0.12$ & $20.88 \pm 0.07$ & $18.55 \pm 0.08$ & $15.80 \pm 0.08$ & $15.05 \pm 0.09$ & $14.75 \pm 0.11$ \\
\hline 23 & WFI J08433843-5246379 & HR-C & & $08: 43: 38.431$ & $-52: 46: 37.92$ & 18.09 & $16.96 \pm 0.08$ & $15.49 \pm 0.03$ & $14.36 \pm 0.04$ & $12.38 \pm 0.02$ & $11.36 \pm 0.02$ & $11.06 \pm 0.02$ \\
\hline 24 & WFI J08433939-5307563 OD & HR-C & $\mathrm{b}$ & 08:43:39.389 & $-53: 07: 56.32$ & 18.38 & $18.08 \pm 0.07$ & $16.87 \pm 0.04$ & $15.20 \pm 0.05$ & $13.14 \pm 0.02$ & $12.54 \pm 0.03$ & $12.28 \pm 0.02$ \\
\hline 25 & WFI J08433945-5248576 & HR-C & & $08: 43: 39.451$ & $-52: 48: 57.56$ & 19.74 & $18.79 \pm 0.07$ & $17.60 \pm 0.03$ & $16.16 \pm 0.04$ & $14.38 \pm 0.03$ & $13.76 \pm 0.03$ & $13.46 \pm 0.05$ \\
\hline 26 & WFI J08434640-5257036 & HR-C, PM-C & & 08:43:46.399 & $-52: 57: 03.56$ & 14.48 & $13.73 \pm 0.06$ & $12.77 \pm 0.03$ & $12.12 \pm 0.04$ & $10.86 \pm 0.03$ & $10.23^{U L}$ & $10.01^{U L}$ \\
\hline 27 & WFI J08434751-5250136 & HR-C, PM-C & & $08: 43: 47.508$ & $-52: 50: 13.63$ & 16.01 & $15.92 \pm 0.07$ & $14.59 \pm 0.03$ & $13.64 \pm 0.03$ & $11.88 \pm 0.02$ & $10.91 \pm 0.02$ & $10.65 \pm 0.02$ \\
\hline 28 & WFI J08434909-5307269 & HR-C & & 08:43:49.090 & $-53: 07: 26.94$ & 19.51 & $18.64 \pm 0.07$ & $17.48 \pm 0.03$ & $16.00 \pm 0.04$ & $14.13 \pm 0.03$ & $13.55 \pm 0.03$ & $13.28 \pm 0.04$ \\
\hline 29 & WFI J08440199-5310356 & HR-C, PM-C & & 08:44:01.992 & $-53: 10: 35.62$ & 15.20 & $14.95 \pm 0.06$ & $13.94 \pm 0.03$ & $13.24 \pm 0.04$ & $11.65 \pm 0.04$ & $11.09 \pm 0.05$ & $10.69 \pm 0.03$ \\
\hline 30 & WFI J08404683-5302487*OD & $\mathrm{M}$ & $\mathrm{c}$ & $08: 40: 46.826$ & $-53: 02: 48.73$ & - & $19.25 \pm 0.06$ & $18.36 \pm 0.03$ & $17.80 \pm 0.04$ & $16.53 \pm 0.21$ & $15.81 \pm 0.28$ & $15.95^{U L}$ \\
\hline 33 & WFI J08412550-5300154* & IR-C & & 08:41:25.502 & $-53: 00: 15.44$ & - & $18.73 \pm 0.05$ & $17.92 \pm 0.04$ & $17.49 \pm 0.05$ & $16.35 \pm 0.11$ & - & $15.60^{U L}$ \\
\hline 34 & WFI J08413601-5309274*OD & M & $\mathrm{d}, \mathrm{e}$ & $08: 41: 36.010$ & $-53: 09: 27.40$ & 19.37 & $18.52 \pm 0.07$ & $17.27 \pm 0.04$ & $15.56 \pm 0.05$ & $13.43 \pm 0.02$ & $12.89 \pm 0.03$ & $12.61 \pm 0.03$ \\
\hline 35 & WFI J08413888-5259492* & IR-C & & $08: 41: 38.885$ & $-52: 59: 49.24$ & 17.22 & $17.20 \pm 0.06$ & $16.30 \pm 0.03$ & $15.73 \pm 0.04$ & $14.56 \pm 0.02$ & $14.16 \pm 0.03$ & $13.95 \pm 0.06$ \\
\hline 36 & WFI J08414904-5255192* & IR-C & & 08:41:49.039 & $-52: 55: 19.20$ & 19.56 & $18.52 \pm 0.06$ & $17.46 \pm 0.03$ & $16.77 \pm 0.04$ & $15.19 \pm 0.05$ & $14.61 \pm 0.06$ & $14.47 \pm 0.10$ \\
\hline 37 & WFI J08415780-5252140*OD & M & $\mathrm{f}$ & 08:41:57.799 & $-52: 52: 13.98$ & 14.74 & $13.76 \pm 0.06$ & $12.74 \pm 0.03$ & $12.16 \pm 0.04$ & $11.09 \pm 0.02$ & $10.40 \pm 0.02$ & $10.27 \pm 0.02$ \\
\hline
\end{tabular}

$\wedge \mathrm{M}=$ confirmed cluster member; HR-C = member candidate according to the location on the HR diagram; PM-C = proper motion member candidate; $\mathrm{X}=\mathrm{X}$-ray sources (Marino et al. 2005).; IR-C: young stellar object candidate according to the IR properties (Sect. 6.1).

- a) Rolleston et al. (1997); b) Patten \& Pavlovski (1999); c) Dodd (2004); d) Barrado y Navascués et al. (2001b); e) Marino et al. (2005); f) Siegler et al. (2007) and references therein.

${ }^{\dagger}$ From the WFI survey presented in this work.

- From the NOMAD catalog (Zacharias et al. 2005). Photometric uncertainties are approximately 0.2-0.3 mag.

¥ From the 2MASS catalog (Cutri et al. 2003).

* Object showing IR excess emission at $24 \mu \mathrm{m}$.

UL Flux upper limit.

OD Other Designation. ID 5: RB729, ID 9: RB955, 2MASSJ08411862-5258546. ID 24: PP9, 2MASSJ08433935-5307556. ID 30: D97, 2MASS J08404679-5302481. ID 32: D112, 2MASSJ08412176-5247291. ID 34: CTIO100, 2MASSJ08413595-5309268. ID 37: V368Vel, SMY29, PMM4636, GSC08569-01100, VXRPSPC41, SHJM9, VXRHRI19, \#29.

\subsection{Statement on the selection results}

Our selection method allowed us to identify 29 new candidate members of IC 2391: 9 selected by photometry and astrometry, and 20 selected by photometry, for which no PM data is available. In Tables 4 and 5, we report the optical and nearIR photometry for these candidates and their stellar parameters as explained in Sect. 3.1; the object designation follows the scheme already adopted by Spezzi et al. (2007). A few of these objects coincide with member candidates selected in previous photometric surveys (Patten \& Pavlovski 1999; Rolleston et al. 1997), as indicated in Table 4 (Col. 4). Moreover, 10 of our candidates, i.e. 34\%, are X-ray sources detected with XMM-EPIC by Marino et al. (2005); these 10 objects are marked with an "X" in Table 4 (Col. 3). We note that the XMM-EPIC pointing in IC 2391 is centered on $\mathrm{RA} \approx 8^{\mathrm{h}} 42^{\mathrm{m}} 00^{\mathrm{s}}$ and $\mathrm{Dec} \approx-53^{\mathrm{d}} 00^{\prime} 36^{\prime \prime}$ and the instrument field of view has a diameter of 30 arcmin. Thus, the vast majority of our candidates are located in the area observed by Marino et al. (2005), and the reason that $66 \%$ of 

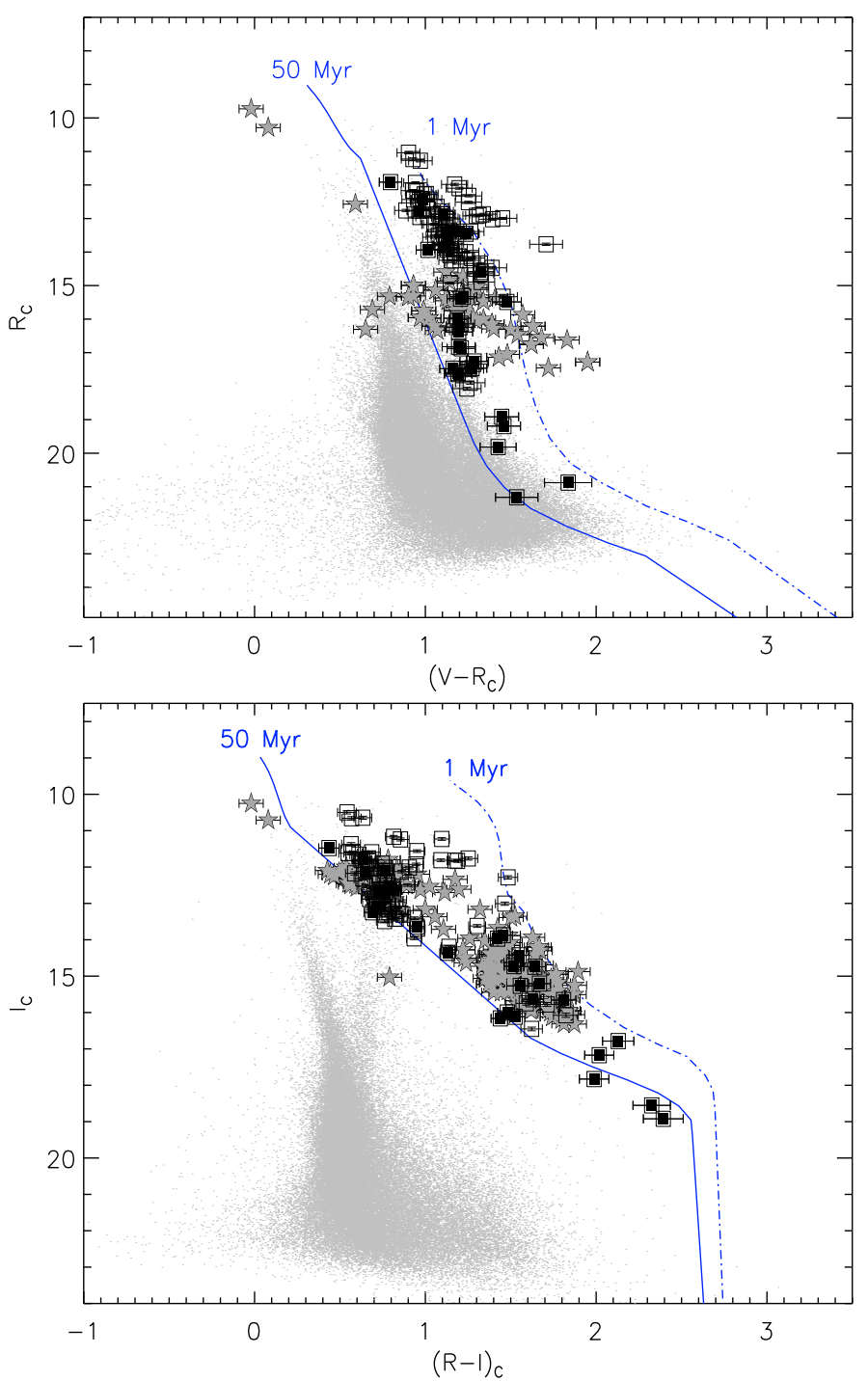

Fig. 6. $R_{\mathrm{C}}$ vs. $\left(V-R_{\mathrm{C}}\right)$ and $I_{\mathrm{C}}$ vs. $(R-I)_{\mathrm{C}} \mathrm{CMDs}$ for the point-like sources in our WFI survey (small gray dots). The lines represent the 1 and 50 Myr theoretical isochrones derived as explained in Sect. 3.3, shifted to the distance modulus of IC 2391 (5.88 mag; Forbes et al. 2001). The open squares represent the sample of optical sources whose isochronal age is $<100 \mathrm{Myr}$ (Sect. 3.2), while the filled squares mark the final sample of 29 member candidates (Sect. 3.3). The stars are the confirmed cluster members, whose photometry was retrieved from the literature (Barrado y Navascués et al. 2001b; Patten \& Simon 1996; Patten \& Pavlovski 1999; Rolleston et al. 1997; Simon \& Patten 1998). Error bars are also drawn; where not visible, they are smaller than the symbol size.

them were not detected must be mainly due to their faint X-ray emission (i.e., below the XMM-EPIC sensitivity limits; Ehle et al. 2008).

In addition, we correctly recovered 32 previously known cluster members. We note that these 32 objects represent the $\sim 40 \%$ of the IC 2391 known population falling in the area observed with WFI; the remaining $60 \%$ of the sources are either saturated in our WFI images or do not have complete $V R_{\mathrm{C}} I_{\mathrm{C}} J H K_{\mathrm{S}}$ photometric data-set in our merged catalog, so we were unable to apply our selection criteria. However, using their magnitudes and proper motions from the the literature (Barrado y Navascués et al. 1999, 2001b, 2004; Cutri et al. 2003; Patten \& Simon 1996; Patten \& Pavlovski 1999; Platais et al. 2007;
Rolleston et al. 1997; Simon \& Patten 1998; Zacharias et al. 2005), they would be found within our selection limits (see Figs. 5 and 6).

All of these arguments support the reliability of our selection method.

\subsection{Multiple visual systems}

To identify possible multiple systems, we visually inspected the 32 IC 2391 members falling in the area observed with WFI and the proposed 29 candidates in all the available images. Following the prescription by Reipurth \& Zinnecker (1993), we perform this search for visual neighbors in the separation range 1.25 arcsec. The lower limit of this range was fixed by considering the typical seeing of our images (Sect. 2.1). The upper limit is imposed to avoid contamination from fore- and background stars. This cut-off is somewhat arbitrary; we can justify this choice by considering that the typical star-to-star separation in our images is $\sim 5$ arcsec and, when a visual companion is found within this distance, it is mostly located conspicuously close to the primary in an otherwise rather empty area of the image.

Twenty-two objects in our sample are found to have neighbors to within less than 5 arcsec. However, a nearby star is accepted to be a possible companion only if it shows indications of youth. Two of these neighbors have a complete $V R_{\mathrm{C}} I_{\mathrm{C}} J H K_{\mathrm{S}}$ photometric data-set but do not fulfill our membership criteria (Sect. 3) and hence were rejected. The remaining 20 neighbors are too faint and do not have near-IR detection in 2MASS, so the only possible indication of youth comes from their position on the optical CMDs. We found that 7 of these 20 neighbors have optical magnitudes and colors consistent or marginally consistent with those of IC 2391 members ${ }^{6}$. These 7 objects, together with their possible primary companion and the relative angular separation, are reported in Table 6 . We note that at the distance of IC 2391 ( $\sim 150 \mathrm{pc})$, the range of angular separation covered by these possible visual binaries corresponds to a linear separation of 350-700 AU, which is reasonable for physically bound young low-mass stars (Reipurth \& Zinnecker 1993). For the member candidate ID 6, two neighbors were found within a 5 arcsec radius; both of these neighbors have optical magnitudes and colors consistent with members of the cluster and hence together these stars might represent a triple system.

\section{Estimating the contamination}

In Sect. 3.3, we adopted the proper motion membership criterion to remove stellar contaminants in the magnitude range $R_{\mathrm{C}} \lesssim 16 \mathrm{mag}$. We could not perform this analysis for fainter candidates because their proper motions are unknown and hence, we still expect a certain level of contamination by field stars in our candidate sample.

To estimate the degree of contamination, we used optical imaging of a nearby field available from the ESO archive. This field was observed with WFI in the VRI filters and is centered on $\mathrm{RA}=08^{\mathrm{h}}: 43^{\mathrm{m}}: 53^{\mathrm{s}}$ and Dec $=-52^{\mathrm{o}}: 47^{\mathrm{m}}: 33^{\mathrm{s}}$, i.e., about $0.9 \mathrm{deg}$ north-east of the center of IC 2391; since the cluster has an apparent diameter of about 50 arcmin (Kharchenko et al. 2005), we expect this area to be more or less devoid of cluster members and to be usable as a control field. The exposure time of the control field images is $600 \mathrm{~s}$ in all the filters; this implies that all the source brighter than $R \approx 15$ are saturated, while sources with $15 \lessgtr R \lessgtr 21$ are detected with a signal-to-noise

${ }^{6}$ This membership criterion is explained in Sect. 3.3. 
Table 5. Estimates of the stellar parameters for the IC 2391 members and candidates of IC 2391 discussed in this work. The objects with identification number (ID) from 1 to 29 are the new candidates selected in this work (Sect. 3); three of these candidates show IR excess emission at $24 \mu \mathrm{m}$ and are marked by an asterisk. The objects with ID from 30 to 37 are the additional 8 cluster members and candidates showing IR excess emission discussed in Sect. 6.

\begin{tabular}{|c|c|c|c|c|c|c|c|}
\hline$\overline{\mathrm{ID}}$ & Designation & $\begin{array}{c}A_{V} \\
(\mathrm{mag})\end{array}$ & $\begin{array}{l}T_{\mathrm{eff}}^{\bullet} \\
(\mathrm{K})\end{array}$ & $\begin{array}{c}L_{\star} \\
\left(L_{\odot}\right)\end{array}$ & $\begin{array}{c}R_{\star} \\
\left(R_{\odot}\right)\end{array}$ & $\begin{array}{c}\text { Mass }^{\dagger} \\
\left(M_{\odot}\right)\end{array}$ & $\begin{array}{l}\text { Age }^{\dagger} \\
(\mathrm{Myr})\end{array}$ \\
\hline 1 & WFI J08403964-5250429* & $1.63 \pm 0.13$ & 3000 & $0.003 \pm 0.001$ & $0.19 \pm 0.04$ & $0.08 \pm 0.02$ & $56 \pm 20$ \\
\hline 2 & WFI J08404512-5248521 & $0.01 \pm 0.06$ & 3400 & $0.038 \pm 0.017$ & $0.56 \pm 0.13$ & $0.25 \pm 0.05$ & $14 \pm 5$ \\
\hline 3 & WFI J08404578-5301052 & $0.97 \pm 0.07$ & 4200 & $0.164 \pm 0.075$ & $0.76 \pm 0.18$ & $0.75 \pm 0.10$ & $45 \pm 20$ \\
\hline 4 & WFI J08405518-5257227 & $1.80 \pm 0.06$ & 4200 & $0.256 \pm 0.118$ & $0.86 \pm 0.22$ & $0.85 \pm 0.10$ & $32 \pm 10$ \\
\hline 5 & WFI J08410806-5300053 & $0.19 \pm 0.10$ & 3100 & $0.015 \pm 0.007$ & $0.43 \pm 0.10$ & $0.07 \pm 0.02$ & $6 \pm 3$ \\
\hline 6 & WFI J08410814-5255027 & $1.96 \pm 0.07$ & 4200 & $0.261 \pm 0.120$ & $0.97 \pm 0.22$ & $0.90 \pm 0.10$ & $25 \pm 8$ \\
\hline 7 & WFI J08410886-5248387* & $0.50 \pm 0.08$ & 3000 & $0.012 \pm 0.006$ & $0.42 \pm 0.09$ & $0.07 \pm 0.02$ & $6 \pm 3$ \\
\hline 8 & WFI J08410937-5302136 & $0.26 \pm 0.07$ & 3100 & $0.024 \pm 0.011$ & $0.54 \pm 0.12$ & $0.13 \pm 0.06$ & $7 \pm 2$ \\
\hline 9 & WFI J08411865-5258549 & $0.05 \pm 0.08$ & 3100 & $0.007 \pm 0.003$ & $0.28 \pm 0.06$ & $0.11 \pm 0.04$ & $32 \pm 10$ \\
\hline 10 & WFI J08412229-5304471 & $0.03 \pm 0.07$ & 3100 & $0.031 \pm 0.014$ & $0.61 \pm 0.14$ & $0.11 \pm 0.04$ & $4 \pm 2$ \\
\hline 11 & WFI J08413933-5252565 & $0.42 \pm 0.06$ & 4000 & $0.093 \pm 0.043$ & $0.64 \pm 0.15$ & $0.62 \pm 0.08$ & $91 \pm 40$ \\
\hline 12 & WFI J08415360-5257558 & $0.21 \pm 0.07$ & 3200 & $0.023 \pm 0.011$ & $0.50 \pm 0.12$ & $0.20 \pm 0.04$ & $18 \pm 6$ \\
\hline 13 & WFI J08422460-5259575 & $0.07 \pm 0.06$ & 4800 & $0.288 \pm 0.133$ & $0.78 \pm 0.18$ & $0.85 \pm 0.10$ & $74 \pm 30$ \\
\hline 14 & WFI J08423071-5257348 & $0.05 \pm 0.06$ & 4000 & $0.102 \pm 0.047$ & $0.67 \pm 0.15$ & $0.70 \pm 0.10$ & $45 \pm 20$ \\
\hline 15 & WFI J08424731-5309235 & $1.74 \pm 0.10$ & 2900 & $0.008 \pm 0.004$ & $0.35 \pm 0.08$ & $0.05 \pm 0.02$ & $6 \pm 3$ \\
\hline 16 & WFI J08424850-5303187 & $0.03 \pm 0.07$ & 3000 & $0.011 \pm 0.005$ & $0.38 \pm 0.09$ & $0.08 \pm 0.02$ & $9 \pm 4$ \\
\hline 17 & WFI J08425873-5305546 & $0.22 \pm 0.06$ & 3300 & $0.049 \pm 0.023$ & $0.68 \pm 0.16$ & $0.25 \pm 0.05$ & $9 \pm 4$ \\
\hline 18 & WFI J08425988-5305074 & $1.74 \pm 0.10$ & 3000 & $0.005 \pm 0.002$ & $0.26 \pm 0.06$ & $0.07 \pm 0.02$ & $18 \pm 6$ \\
\hline 19 & WFI J08430294-5302259 & $0.63 \pm 0.06$ & 4000 & $0.252 \pm 0.116$ & $1.05 \pm 0.24$ & $0.90 \pm 0.10$ & $18 \pm 6$ \\
\hline 20 & WFI J08431228-5251411 & $3.19 \pm 0.19$ & 2900 & $0.002 \pm 0.001$ & $0.18 \pm 0.04$ & $0.07 \pm 0.02$ & $64 \pm 20$ \\
\hline 21 & WFI J08431860-5306241 & $0.18 \pm 0.06$ & 4200 & $0.253 \pm 0.117$ & $0.95 \pm 0.22$ & $0.85 \pm 0.10$ & $32 \pm 10$ \\
\hline 22 & WFI J08433366-5259549 & $3.51 \pm 0.16$ & 3000 & $0.003 \pm 0.001$ & $0.20 \pm 0.05$ & $0.09 \pm 0.02$ & $64 \pm 20$ \\
\hline 23 & WFI J08433843-5246379 & $2.53 \pm 0.06$ & 3900 & $0.076 \pm 0.035$ & $0.61 \pm 0.14$ & $0.60 \pm 0.08$ & $90 \pm 40$ \\
\hline 24 & WFI J08433939-5307563 & $0.02 \pm 0.07$ & 3000 & $0.016 \pm 0.007$ & $0.47 \pm 0.11$ & $0.08 \pm 0.02$ & $6 \pm 3$ \\
\hline 25 & WFI J08433945-5248576 & $0.12 \pm 0.08$ & 3300 & $0.006 \pm 0.003$ & $0.23 \pm 0.05$ & $0.15 \pm 0.04$ & $80 \pm 40$ \\
\hline 26 & WFI J08434640-5257036 & $0.12 \pm 0.10$ & 4200 & $0.180 \pm 0.083$ & $0.80 \pm 0.19$ & $0.75 \pm 0.08$ & $50 \pm 20$ \\
\hline 27 & WFI J08434751-5250136 & $2.00 \pm 0.06$ & 4000 & $0.110 \pm 0.051$ & $0.69 \pm 0.16$ & $0.70 \pm 0.08$ & $45 \pm 20$ \\
\hline 28 & WFI J08434909-5307269 & $0.01 \pm 0.08$ & 3200 & $0.007 \pm 0.003$ & $0.26 \pm 0.06$ & $0.11 \pm 0.03$ & $32 \pm 10$ \\
\hline 29 & WFI J08440199-5310356 & $0.41 \pm 0.07$ & 4000 & $0.083 \pm 0.038$ & $0.60 \pm 0.14$ & $0.62 \pm 0.08$ & $80 \pm 40$ \\
\hline 30 & WFI J08404683-5302487* & $0.16 \pm 0.17$ & 4200 & $2.128 \pm 0.001$ & $2.77 \pm 0.05$ & $1.40 \pm 0.20$ & $5 \pm 2$ \\
\hline 31 & WFI J08410019-5249088* & $0.77^{\ddagger}$ & $3800^{\ddagger}$ & $0.185^{\ddagger}$ & $0.97^{+}$ & $0.75^{\ddagger}$ & $20^{\ddagger}$ \\
\hline 32 & WFI J08412176-5247289* & $0.22 \pm 0.15$ & 4300 & $1.087 \pm 0.001$ & $1.80 \pm 0.01$ & $1.40 \pm 0.20$ & $6 \pm 3$ \\
\hline 33 & WFI J08412550-5300154* & $0.13^{\ddagger}$ & $4300^{\ddagger}$ & $0.700^{\ddagger}$ & $2.48^{\ddagger}$ & $1.20^{\ddagger}$ & $10^{\ddagger}$ \\
\hline 34 & WFI J08413601-5309274* & $0.17 \pm 0.07$ & 3000 & $0.012 \pm 0.006$ & $0.39 \pm 0.09$ & $0.07 \pm 0.02$ & $7 \pm 3$ \\
\hline 35 & WFI J08413888-5259492* & $0.44^{\ddagger}$ & $4100^{\ddagger}$ & $0.101^{\ddagger}$ & $1.13^{\ddagger}$ & $0.65^{\ddagger}$ & $80^{\ddagger}$ \\
\hline 36 & WFI J08414904-5255192* & $0.05^{+}$ & $3700^{\ddagger}$ & $0.127^{\ddagger}$ & $0.95^{\ddagger}$ & $0.65^{+}$ & $16^{+}$ \\
\hline 37 & WFI J08415780-5252140* & $0.24 \pm 0.2$ & 4000 & $0.203 \pm 0.069$ & $0.74 \pm 0.16$ & $0.85 \pm 0.10$ & $25 \pm 8$ \\
\hline
\end{tabular}

- The uncertainty on $T_{\text {eff }}$ is assumed to be $250 \mathrm{~K}$ on average (see Sect. 3.1).

$\dagger$ From the evolutionary models by Baraffe et al. (1998) and Chabrier et al. (2000).

* From the Robitaille et al. (2006) models for young stellar objects (see Sect. 6.1.1).

* Object showing IR excess emission at $24 \mu \mathrm{m}$.

ratio of higher than 10 . Thus, using these data, we could estimate the level of contamination at least in the photometric range spanned by our candidates with no proper motion determination. By using the same procedure outlined in Sect. 3, we found $12 \mathrm{ob}-$ jects in our control field satisfying our photometric cluster membership criteria. Therefore, we estimate that the 17 objects with $15 \lesssim R \lesssim 21$ cataloged by us to be candidate cluster members (Table 4 ), still have a probability up to $\sim 70 \%$ of being spurious.

To verify further the amount of expected contamination in all the photometric range covered by our candidates, i.e., $12 \lesssim R_{\mathrm{C}} \lesssim$ 21 , we have also used a different approach. Nearly all members of IC 2391 are located in the region of the HR diagram defined approximately by the $10 \mathrm{Myr}$ and $100 \mathrm{Myr}$ isochrones (Fig. 5). In particular, all our candidates are confined within the region defined by $T_{\text {eff }}=2900-4800 \mathrm{~K}$ and $L_{\star}=0.002-0.4 L_{\odot}$ (i.e., absolute visual magnitude $M_{V}=12-6 \mathrm{mag}$ ); thus, to estimate the contamination level of our candidate sample, we must estimate the numbers of stars unrelated to the cluster that may appear in this region of the HR diagram. The number of interloping stars can be probed by using analytic models of the Galactic stellar distribution, i.e., simulations of the expected properties of stars seen towards a given direction of the Galaxy over a given solid angle. We performed this exercise by using the Galaxy model by Robin et al. (2003) and their online tool ${ }^{7}$. In the temperature range of our candidates, foreground stars are expected to

\footnotetext{
7 For details on the adopted Galaxy model, we refer the reader to Robin et al. (2003). The online simulation tool is available at: http://bison.obs-besancon.fr/modele/
} 
Table 6. Possible multiple systems in IC 2391 (see Sect. 3.5).

\begin{tabular}{|c|c|c|c|c|c|c|c|c|c|}
\hline & & Primary star & & Separation & & & Neighbor star & & \\
\hline & ID & Designation & Ref. $^{\dagger}$ & $(\operatorname{arcsec})$ & RA & Dec & $V$ & $R_{\mathrm{c}}$ & $I_{\mathrm{c}}$ \\
\hline \multirow{2}{*}{$\begin{array}{l}\text { Previously known } \\
\text { cluster members }\end{array}$} & - & Cl* IC 2391 CTIO 126 & $a$ & 3.1 & $08: 42: 49.385$ & $-52: 52: 16.96$ & $18.25 \pm 0.07$ & $17.13 \pm 0.03$ & $16.46 \pm 0.04$ \\
\hline & - & $\mathrm{Cl}^{*}$ IC 2391 CTIO 25 & $a$ & 2.8 & $08: 42: 46.025$ & $-52: 46: 52.64$ & $20.02 \pm 0.07$ & $18.82 \pm 0.03$ & $17.88 \pm 0.06$ \\
\hline \multirow{4}{*}{$\begin{array}{l}\text { New member } \\
\text { candidates }\end{array}$} & " & $" \prime$ & $b$ & 3.5 & 08:41:08.489 & $-52: 55: 04.19$ & $15.72 \pm 0.05$ & $15.02 \pm 0.04$ & $14.70 \pm 0.05$ \\
\hline & 26 & WFI J08434640-5257036 & $b$ & 4.5 & $08: 43: 46.814$ & $-52: 57: 06.12$ & $16.39 \pm 0.05$ & $15.68 \pm 0.04$ & $15.26 \pm 0.05$ \\
\hline & 27 & WFI J08434751-5250136 & $b$ & 3.3 & $08: 43: 47.844$ & $-52: 50: 12.48$ & $20.76 \pm 0.07$ & $19.45 \pm 0.03$ & $18.52 \pm 0.04$ \\
\hline & 29 & WFI J08440199-5310356 & $b$ & 2.4 & $08: 44: 02.071$ & $-53: 10: 37.92$ & $15.48 \pm 0.06$ & $14.47 \pm 0.03$ & $13.77 \pm 0.04$ \\
\hline
\end{tabular}

$\dagger{ }^{a}$ Barrado y Navascués et al. (2001b); ${ }^{b}$ This work.

be main-sequence cool dwarfs, whereas red giants are expected to dominate the background population. Assuming a cluster distance of 150-200 pc, we expect some 14 foreground dwarfs in the $\sim 30 \times 30$ square arcmin area observed in IC 2391 with apparent $R_{\mathrm{C}}$ magnitude between 12 and 21 and spectral types of MK, i.e., the ranges estimated for our candidates (see Tables 4 and 5). No background giants are expected to be found in the locus occupied by the cluster members because all these stars appear much brighter than IC 2391 members in the same effective temperature range. We thus conclude that only foreground cool main-sequence stars can contribute noticeably to the contamination of our candidate sample, with the expected contamination level being $48 \%$. We note that this value is very close to the pollution rate of $50 \%$ estimated by Barrado y Navascués et al. (2001b) for the same area in IC 2391.

Another possible source of contamination is represented by galaxies that may have colors similar to those of PMS objects. However, these bright galaxies are a priori excluded from our selection, because our PSF extraction method allows an efficient identification/removal of extended objects (Sect. 2.1.4). The only source of contamination may be point-like extragalactic objects, i.e., QSO's. According to QSO's number counts (Fontanot et al. 2007; Richards et al. 2005), the predicted number of QSO's per square degree become significant (i.e., greater than $\sim 10 / \mathrm{deg}^{2}$ ) at magnitudes fainter than $V \approx 22$, while the majority of our candidates are brighter. Thus, extra-galactic objects are likely to affect our candidate sample by no more than $3 \%$.

In conclusion, the expected contamination of our candidate sample due to field objects is $\sim 50 \%$.

\section{On the sub-stellar population in IC 2391}

The first and only estimate of the IC 2391 mass spectrum in the very low-mass domain was derived by Barrado y Navascués et al. (2004). Independently of the assumed cluster age of between 25 and $50 \mathrm{Myr}$, they found an index of the power-law mass function in the mass range $0.072-0.5 M_{\odot}$ equal to $0.96 \pm 0.12$, consistent with the value reported for other open clusters (e.g. Barrado y Navascués et al. 2001a, 2002; Barrado y Navascués \& Stauffer 2003; Bouvier et al. 1998). Below $0.07 M_{\odot}$, the cluster mass spectrum presents a sudden drop which these authors partially attribute to the lack of completeness of their survey at magnitudes fainter than $I \approx 18.5$ (i.e. $0.05 M_{\odot}$ for cluster members).

Although our survey is complete at the $100 \%$ and $80 \%$ levels down to $0.05 M_{\odot}$ and $0.03 M_{\odot}$, respectively, and covers $60 \%$ of the sky area spanned by the cluster, we found only an handful of candidate substellar members. In terms of numbers, considering the IC 2391 populations observed before to January 2008 (i.e., 180 objects; Barrado y Navascués et al. 1999, 2001b, 2004; Patten \& Simon 1996; Patten \& Pavlovski 1999; Platais et al. 2007; Rolleston et al. 1997; Simon \& Patten 1998), there are 14 members with $T_{\text {eff }} \lesssim 3100 \mathrm{~K}$, i.e., below the approximate substellar limit for 30-50 Myr old objects. Thus, the fraction of sub stellar objects, $R_{\mathrm{Ss}}=\frac{N\left(0.02 M_{\odot}-0.08 M_{\odot}\right)}{N\left(0.08 M_{\odot}-10 M_{\odot}\right)}$, in this cluster is $\sim 8 \%$; by adding our $12 \mathrm{BD}$ candidates (i.e. candidates with $T_{\text {eff }} \leq 3100 \mathrm{~K}$; see Table 5), this fraction would increase to $\sim 15 \%$. However, all our BD candidates have $R$-magnitudes in the range $15 \lesssim R \lesssim 21$, where we expect a high level of contamination (70\%, see Sect. 4). Thus, the true value of $R_{\mathrm{ss}}$ is expected to be close to $10 \%$. Our physical parametrization also indicates that all our BD candidates have $T_{\text {eff }} \gtrsim 2900 \mathrm{~K}$ and masses very close to the hydrogen-burning limit. Thus, the results of our deeper optical survey confirm the lack of members with spectral type later than M7-M8 in IC 2391 found earlier by Barrado y Navascués et al. (2004). A similar result was obtained for the nearly coeval open clusters $\alpha$ Per ( 50 Myr) and NGC 2547 ( 25 Myr) by Barrado y Navascués et al. (2002) and Jeffries et al. (2004), respectively. Thus, as already concluded by Barrado y Navascués et al. (2004), one of the most solid hypothesis explaining the BD deficit in IC 2391 and other open clusters remains that by Dobbie et al. (2002) and Jameson et al. (2003), i.e., the onset of larger-size dust grain formation in the upper atmosphere of objects with spectral types M7-M8 or later, which would be responsible of the local drop in the shape of the luminosity-mass relation.

Of course, a number of alternative hypotheses might also be invoked. For instance, simulations of the dynamics of BD populations in open clusters, which consider the effects of a large range of primordial binary populations, also demonstrated that the majority of BDs might be contained within primordial binary systems, which then prevent a large proportion of them from being detected (Adams et al. 2002). Alternatively, the deficit of BDs in IC 2391 might be due to mass segregation and/or evaporation effects. Dynamical mass segregation acts on a timescale similar to the relaxation time of a cluster (Bonnell \& Davies 1998), which for clusters of the size of IC 2391 is on the order of 1 Myr (Binney \& Tremaine 1987, p. 390). Sagar \& Bhatt (1989) also found indication that the higher mass stars in IC 2391 have lower velocity dispersions than the lower mass stars.

\section{On the debris disk population in IC 2391}

The first survey for debris disks in IC 2391 was performed by Siegler et al. (2007) using MIPS on board Spitzer. The central square degree of IC 2391 was observed at $24 \mu \mathrm{m}$, with the aim of studying the incidence of debris disks in the cluster. The authors reported detecting an IR excess indicative of debris disks around 8 previously known members of the cluster, namely 1 A star, 6 FGK stars and $1 \mathrm{M}$ dwarf, and estimated a debris disk fraction of $10 \%$ for BA stars and $31 \%$ for FGK stars. 
We investigate further the debris disk population in IC 2391, using our optical data in combination with 2MASS data, the MIPS catalog by Siegler et al. (2007), and additional IR data from IRAC. This dataset is suitable for probing disk radii from $\sim 0.1$ AU up to $20 \mathrm{AU}$ depending on the stellar mass (Merín et al. in preparation). While the work by Siegler et al. (2007) focuses only on the previously known cluster members, we extend the search for objects with IR excess emission to all sources detected at $24 \mu \mathrm{m}$ that have a counterpart in our optical WFI images. This allow us to identify new candidate KM-type members of the cluster with debris disks. We remind the reader that our search is spatially limited to the overlapping area observed with WFI and Spitzer (Fig. 1). The selected debris disk candidates should also be investigated spectroscopically to assess definitively their membership to the cluster. The experience gained in analyzing the Spitzer Core to Disk Legacy Survey (hereafter c2d; Evans et al. 2003) implies that samples of objects showing mid-IR excess in star-forming regions could be highly contaminated by background post-main sequence stars, mostly in the direction of the Galactic plane. This contamination might account for up to $30 \%$ of those objects and could be even higher in the case of IC 2391 , which has a Galactic latitude $\left(b \approx-7^{\circ}\right)$ lower than the typical c $2 \mathrm{~d}$ fields. For all these reasons, our search is intended to provide a deeper insight into the candidate debris disk population in IC 2391.

\subsection{Selection of debris disk candidates}

We identified possible debris disk objects in IC 2391 by measuring $24 \mu \mathrm{m}$ flux densities in excess of the expected photospheric emission; this criterion was adopted in the pioneering study by Siegler et al. (2007) and allows us to select new debris disk candidates in a homogeneous way. We used the $\left(V-K_{\mathrm{S}}\right)$ vs. $\left(K_{\mathrm{S}}-[24 \mu \mathrm{m}]\right)$ color-color $(\mathrm{CC})$ diagram to identify these excess sources across a broad range of spectral types. As demonstrated by Siegler et al. (2007), the $\left(V-K_{\mathrm{S}}\right)$ color is a good tracer for spectral types down to late M-dwarfs, while the $\left(K_{\mathrm{S}}-[24 \mu \mathrm{m}]\right)$ color is a good diagnostic for mid-IR excess because the $K_{\mathrm{S}^{-}}$ band flux is almost entirely photospheric for stars older than $\sim 10 \mathrm{Myr}$, which have already dissipated the innermost region of their disks (Haish et al. 2001). We dereddened the magnitudes of all the sources detected at $24 \mu \mathrm{m}$ using the $A_{V}$ values derived in Sect. 3.1 and the extinction law by Cardelli et al. (1989) ${ }^{8}$. We selected objects with IR excesses at $24 \mu \mathrm{m}$ using the mean dereddened photospheric locus in the $\left(V-K_{\mathrm{S}}\right)$ vs. $\left(K_{\mathrm{S}}-[24 \mu \mathrm{m}]\right)$ diagram determined by Siegler et al. (2007); for spectral types later than M4, we used the IR photospheric colors for M-dwarfs determined by Gautier et al. (2007).

As shown in Fig. 7, we found 10 sources redder than this threshold, i.e., objects possessing $\left(K_{\mathrm{S}}-[24 \mu \mathrm{m}]\right)$ flux ratios in excess of the expected photospheric colors by at least $15 \%$, i.e., the same criterion as in Siegler et al. (2007). Four of these 10 sources (ID 30, ID 32, ID 34, and ID 37), are previously known members of the cluster ${ }^{9}$ (stars in Fig. 7), two (ID 1 and ID 7) are new candidate members selected in this work (squares in Fig. 7), while the remaining four (ID 31, ID 33, ID 35 and ID 36) are new sources with IR excess emission neither reported by previous studies nor selected as candidate cluster members by our optical criteria (crosses in Fig. 7); the latter objects are discussed further in Sect. 6.1.1.

\footnotetext{
8 We adopt the Cardelli et al. (1989) extinction law for consistency with our parametrization procedure (Sect. 3.1).

${ }^{9}$ See Table 4 for previous bibliography on these objects.
}

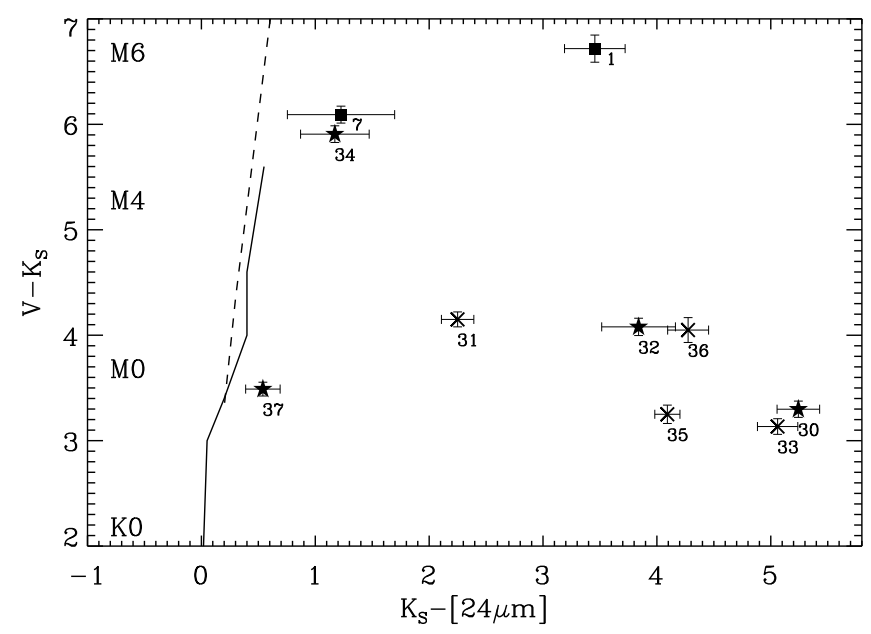

Fig. 7. $\left(V-K_{\mathrm{S}}\right)$ vs. $\left(K_{\mathrm{S}}-[24 \mu \mathrm{m}]\right)$ color-color diagram for the 10 sources in IC 2391 showing IR excess at $24 \mu \mathrm{m}$. The continuous and dashed lines are the mean dereddened photospheric loci determined by Siegler et al. (2007) and Gautier et al. (2007), respectively. Sources redder than this threshold possess $\left(K_{\mathrm{S}}-[24 \mu \mathrm{m}]\right)$ flux ratios in excess of expected photospheric colors. Symbols are as follows: the stars mark the confirmed cluster members, while squares and crosses mark the member candidates and the four sources of dubious nature discussed in Sect. 6.1.1, respectively.

The optical and near-IR photometry and the physical parameters of the 10 sources showing IR excesses at MIPS/24 $\mu \mathrm{m}$ are reported in Tables 4 and 5, respectively, while Table 7 provides their IRAC and MIPS/24 $\mu \mathrm{m}$ magnitudes.

\subsubsection{Comments on individual objects}

Siegler et al. (2007) reported IR excesses at $24 \mu \mathrm{m}$ indicative of debris disks for 8 objects in IC 2391. Only two of these objects are within the area observed with WFI, namely ID 29 and ID 24 from their Table 2; we correctly recovered ID 29 as a possible debris disk object (i.e., our ID 37), while ID 24 is saturated in our WFI images and was then a priori excluded from our analysis.

Four of our debris disk candidates, namely ID 31, ID 33, ID 35, and ID 36, were not selected as candidate cluster members either by previous surveys or by our optical criteria, even though they exhibit a clear IR excess emission in their SEDs (Fig. 8). This is because these four sources are sub-luminous in the HR diagram when the distance of $150 \mathrm{pc}$ is adopted and the extinction is determined as explained in Sect. 3.1. Possible reasons for this inconsistency may be an underestimate of the extinction and/or the distance for these four sources. From a visual inspection of our optical WFI images, these four sources appear point-like and are concentrated in the central area of the cluster (Fig. 1). Moreover, by fitting their SEDs with the Robitaille et al. (2006) grid of models for young stellar objects normalized to the observed $I$-band flux, we obtain stellar and disk parameters that remain consistent with young stars belonging to the cluster (see Table 5). Thus, for the time being, we include these sources in our list of possible debris disk objects in IC 2391; future followup spectroscopy will shed further light on their nature.

\subsection{On the disk properties}

We describe the circumstellar material around the 10 objects with IR excess emission selected above, to study the nature and evolutionary phase of their disks. Analysis of Spitzer 

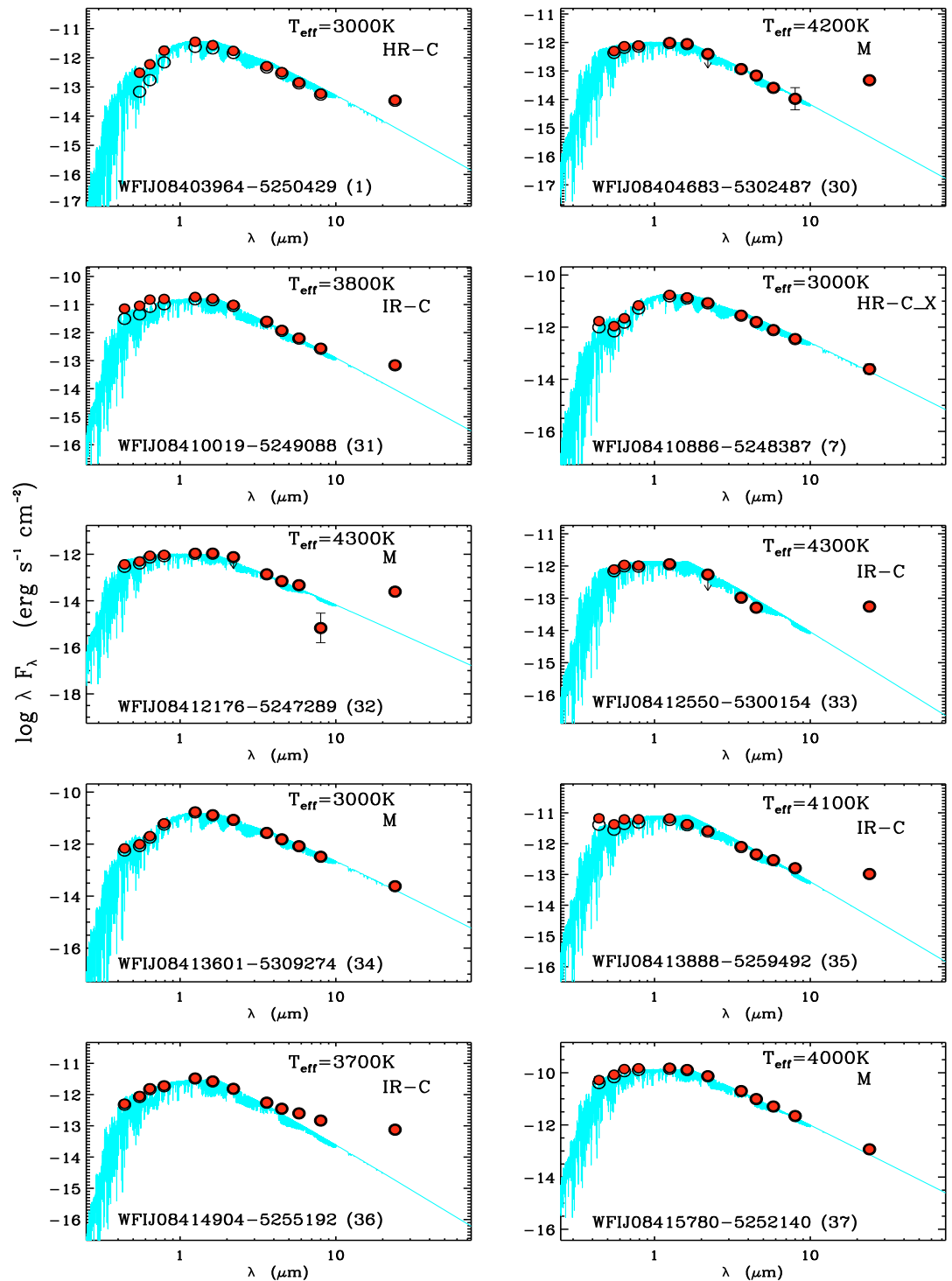

Fig. 8. Spectral energy distributions of the 10 sources in IC 2391 showing IR excess emission identified in this work. The dereddened fluxes are represented with filled circles, while the observed fluxes are represented with open circles. Flux upper limits are marked with an arrow. The best-fit NextGen spectrum by Hauschildt et al. (1999), for objects with $T_{\text {eff }}>4000 \mathrm{~K}$, or StarDusty spectrum by Allard et al. (2000), for objects with $T_{\text {eff }} \leq 4000 \mathrm{~K}$, is overplotted on each SED, representing the stellar emission. Each panel is labeled with the object name (and ID number between brackets), estimated effective temperature (Table 5) and membership status (see Table 7).

Table 7. IRAC and MIPS/24 $\mu \mathrm{m}$ magnitudes and SED slope $\left(\alpha_{[K \& 24 \mu \mathrm{m}]}\right)$ for the 10 objects showing IR excess emission at $24 \mu \mathrm{m}$ identified in this work.

\begin{tabular}{ccccccccc}
\hline \hline ID & Designation & Status $^{\wedge}$ & IRAC/3.6 $\mu \mathrm{m}$ & IRAC/4.5 $\mu \mathrm{m}$ & IRAC/5.8 $\mu \mathrm{m}$ & IRAC/8.0 $\mu \mathrm{m}$ & MIPS/24 $\mu \mathrm{m}$ & $\alpha_{\left[K_{\mathrm{S}} \& 24 \mu \mathrm{m}\right]}$ \\
\hline 1 & WFI J08403964-5250429 & HR-C & $14.26 \pm 0.01$ & $14.02 \pm 0.01$ & $14.13 \pm 0.08$ & $14.11 \pm 0.13$ & $11.07 \pm 0.25$ & -1.63 \\
30 & WFI J08404683-5302487 & M & $15.76 \pm 0.04$ & $15.61 \pm 0.06$ & $15.92 \pm 0.34$ & $15.90 \pm 0.98$ & $10.71 \pm 0.18$ & -0.89 \\
31 & WFI J08410019-5249088 & IR-C & $12.46 \pm 0.01$ & $12.54 \pm 0.01$ & $12.47 \pm 0.02$ & $12.39 \pm 0.04$ & $10.31 \pm 0.14$ & -2.07 \\
7 & WFI J08410886-5248387 & HR-C, X & $12.32 \pm 0.01$ & $12.21 \pm 0.01$ & $12.22 \pm 0.02$ & $12.10 \pm 0.03$ & $11.42 \pm 0.47$ & -2.45 \\
32 & WFI J08412176-5247289 & M & $15.58 \pm 0.03$ & $15.59 \pm 0.05$ & $15.26 \pm 0.23$ & $16.88 \pm 1.60$ & $11.41 \pm 0.32$ & -1.44 \\
33 & WFI J08412550-5300154 & IR-C & $15.88 \pm 0.05$ & $15.93 \pm 0.06$ & - & - & $10.54 \pm 0.17$ & -0.96 \\
34 & WFI J08413601-5309274 & M & $12.35 \pm 0.01$ & $12.24 \pm 0.01$ & $12.14 \pm 0.02$ & $12.17 \pm 0.03$ & $11.44 \pm 0.30$ & -2.46 \\
35 & WFI J08413888-5259492 & IR-C & $13.70 \pm 0.01$ & $13.58 \pm 0.02$ & $13.28 \pm 0.05$ & $12.95 \pm 0.06$ & $9.86 \pm 0.09$ & -1.34 \\
36 & WFI J08414904-5255192 & IR-C & $14.07 \pm 0.02$ & $13.83 \pm 0.02$ & $13.44 \pm 0.06$ & $13.03 \pm 0.06$ & $10.20 \pm 0.15$ & -1.26 \\
37 & WFI J08415780-5252140 & M & $10.18 \pm 0.01$ & $10.22 \pm 0.01$ & $10.17 \pm 0.01$ & $10.10 \pm 0.01$ & $9.73 \pm 0.15$ & -2.71 \\
\hline
\end{tabular}

$\wedge \mathrm{M}=$ confirmed cluster member; HR-C = member candidate according to the location on the HR diagram; $\mathrm{X}=\mathrm{X}$-ray sources (Marino et al. 2005); IR-C: young stellar object candidate according to the IR properties (Sect. 6.1).

${ }^{\dagger}$ Flux at 5.8 and $8 \mu \mathrm{m}$ not available. 
observations in star-forming regions within the framework of the c2d survey (e.g. Alcalá et al. 2008; Harvey et al. 2007; Merín et al. 2008) have provided several methods to study disk properties, such as the amount of circumstellar dust and morphology. We applied some of these methods to investigate the nature of our 10 debris disk candidates in IC 2391.

We first constructed the SEDs of these objects from optical to mid-IR wavelengths, as already described in Sect. 3.1 but including IRAC and MIPS data and, where possible, additional $B$ magnitudes from the NOMAD catalog (Zacharias et al. 2005). For each object, the flux at each wavelength was corrected for interstellar extinction using the $A_{V}$ values reported in Table 5 and the normal $\left(R_{V}=3.1\right)$ reddening law by Cardelli et al. (1989). The SEDs of the 10 debris disk candidates in IC 2391 are shown in Fig. 8 together with the best-fit model spectra by Hauschildt et al. (1999) and Allard et al. (2000) for the same temperature as the objects (see Sect. 3.1), which represents the stellar contribution. For the majority of objects, the IR excess with respect to the expected photospheric emission is clearly visible. Moreover, the lack of near-IR excess emission is indicative of disks whose inner part has already been dissipated (see, e.g., Sicilia-Aguilar et al. 2006).

Additional clues about the nature of our debris disk candidates can be obtained by investigating the objects IR classes according to the definition by Lada (1987) as extended by Greene et al. (1994). This classification is traditionally based on the value of the $\alpha$ spectral index, which is determined as the slope of the SED in the form $\log \lambda F_{\lambda}$ vs. $\log \lambda$ at wavelengths longer than $2 \mu \mathrm{m}$. Following the c $2 \mathrm{~d}$ convention (e.g. Alcalá et al. 2008; Harvey et al. 2007; Merín et al. 2008), we used $\alpha_{[K \& 24 \mu \mathrm{m}]}$ as the reference spectral index, which is the slope of the linear fit to the fluxes at $K_{S}$ and MIPS/24 $\mu$ m (Table 7). As shown in Fig. 9, we found that 5 of the 10 debris disk candidates in our sample exhibit $\alpha_{[K \& 24 \mu \mathrm{m}]}$ slopes typical of IR class III sources $\left(\alpha_{[K \& 24 \mu \mathrm{m}]}<-1.6\right)$ according to the classification by Greene et al. (1994), i.e., they have optically thin disks. The remaining 5 objects fall in the class II regime $\left(-1.6<\alpha_{[K \& 24 \mu \mathrm{m}]} \leq-0.3\right)$, i.e., they should have optically thick disks, but the majority of them lay very close to the class III boundary (i.e. $\alpha_{[K \& 24 \mu \mathrm{m}]} \lesssim$ $-1)$. The possibility of IC 2391 having any thick/transitional disks is quite surprising considering its age (30-50 Myr), because these disks, along with primordial disks, are typically dissipated within 10 Myr (Decin et al. 2003; Dominik \& Decin 2003). However, Spitzer data have shown a clear mass dependence of the disk dissipation timescale, i.e., very low-mass stars dissipate their primordial disks more slowly than more massive stars (Hillenbrand 2008; Scholz et al. 2007).

In conclusion, the 10 sources with IR excess emission selected in Sect. 6.1 are likely to possess evolved disks, whose inner part might have already been cleared by the formation of large-sized bodies (such as rocks).

\section{Conclusions}

We have performed a deep wide-field imaging survey of the young open cluster IC 2391 to study its low mass population down to $0.03 M_{\odot}$. We have used our optical VRI data, in combination with $J H K_{\mathrm{S}}$ magnitudes from the 2MASS catalog to estimate the physical parameters of the optically detected sources and selected cluster-member candidates on the basis of their location in the HR diagram relative to theoretical isochrones. We have estimated the contamination using statistical arguments and, when possible, proper motions from the NOMAD catalog. Our survey has a completeness level of $80 \%$ down to $I_{C}=23.0$,

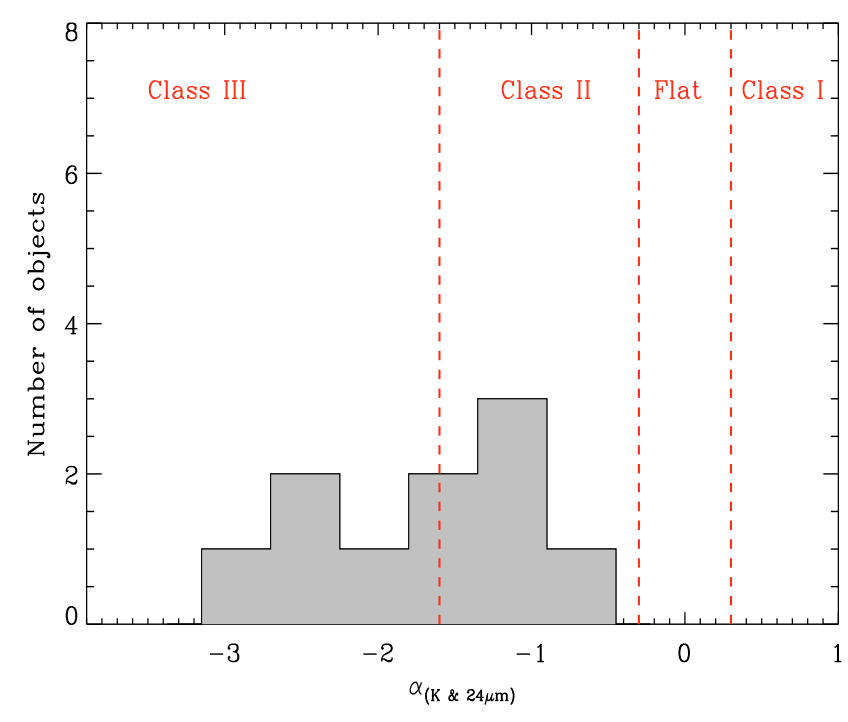

Fig. 9. Distribution of the $\alpha_{[K \& 24 \mu \mathrm{m}]}$ SED slopes for the sources in IC 2391 showing IR excess emission identified in this work. The dashed vertical lines mark the boundaries of class I, flat, class II and class III objects according to the classification by Lada (1987) as extended by Greene et al. (1994)

i.e., $\sim 1-2$ mag fainter than previous optical surveys conducted in IC 2391, and has revealed 29 new low-mass member candidates, among which 12 may be BDs. The expected contaminations is at least $\sim 50 \%$.

We have confirmed the presence of a significant drop in the cluster mass spectrum across the stellar/substellar boundary, the fraction of substellar objects being only $8-15 \%$. The lack of BDs in IC 2391 might be due to the dip in the luminosity function around M7-M8 that has already been observed in other starforming regions, open clusters, and the field, which is caused by the onset of larger-size dust formation in the atmospheres of objects in this effective temperature regime. However, other explanations such as dynamical mass segregation and/or masking in binary systems might be invoked. Our data are unable to help us distinguish between these scenarios.

Finally, we have combined our ground-based optical observations with near-IR photometry from 2MASS and Spitzer GTO observations to investigate the debris disk population of IC 2391. We identified 10 possible debris disk objects in the cluster on the basis of their excess $24 \mu \mathrm{m}$ flux densities relative to their expected photospheric emission. We constructed optical/IR SEDs for all of these objects and investigated the properties of their circumstellar material. According to our analysis, these $10 \mathrm{ob}-$ jects are likely to possess evolved disks whose inner parts have already been cleared. A few of these objects might possess thick or transition disks, whihc would be surprising given the age of IC 2391.

Acknowledgements. This paper is based on observations carried out at the European Southern Observatory, La Silla (Chile), under observing program number 68.D-0541(A). This publication makes use of data products from the Two Micron All Sky Survey, which is a joint project of the University of Massachusetts and the Infrared Processing and Analysis Center/California Institute of Technology, funded by NASA and the National Science Foundation. We also acknowledge extensive use of the SIMBAD database, operated at CDS Strasbourg, the WEBDA database, operated at the Institute for Astronomy of the University of Vienna, and the NOMAD catalog, released by the U.S. Naval 
Observatory. L. Spezzi acknowledges financial support from INAF-Catania. We thank J.M. Alcalá, A. Frasca, D. Gandolfi and F. Comerón for many discussions and suggestions. We are also grateful to many others, in particular to Salvatore Spezzi.

\section{References}

Adams, T., Davies, M. B., Jameson, R. F., \& Scally A. 2002, MNRAS, 333, 547 Alcalá, J. M., Spezzi, L., Chapman, N., et al. 2008, ApJ, 676, 427

Allard, F., Hauschildt, P. H., \& Schwenke, D. 2000, ApJ, 540, 1005

Baraffe, I., Chabrier, G., Allard, F., \& Hauschildt, P. H. 1998, A\&A, 337, 403

Barrado y Navascués, D., Stauffer, J. R., \& Patten, B. M. 1999, ApJ, 522, L53

Barrado y Navascués, D., Bouvier, J., Stauffer, J. R., \& Martìn, E. L. 2001a, ApJ, 546, 1006

Barrado y Navascués, D., Stauffer, J. R., Bricenõ, C., et al. 2001b, ApJS, 134, 103

Barrado y Navascués, D., Bouvier, J., Stauffer, J. R., et al. 2002, A\&A, 395, 813

Barrado y Navascués, D., \& Stauffer, J. R. 2003, in Brown Dwarfs, ed. E. L. Martìn (San Francisco: ASP), IAU Symp., 211, 155

Barrado y Navascués, D., Stauffer, J. R., \& Jayawardhana, R. 2004, ApJ, 614, 386

Basri, G., Marcy, G. W., \& Graham, J. R. 1996, ApJ, 458, 600

Bertin, E., \& Arnouts, S. 1996, A\&AS, 117, 393

Binney, J., \& Tremaine, S. 1987, Galactic Dynamics (Princeton: Princeton Univ. Press)

Bonnell, I. A., \& Davies, M. B. 1998, MNRAS, 295, 691

Bouvier, J., Stauffer, J. R., Martìn, E. L., et al. 1998, A\&A, 336, 490

Bouvier, J., Moraux, E., \& Stauffer, J.R. 2005, ASSL, 327, 61

Cardelli, J. A., Clayton, G. C., \& Mathis, J. S. 1989, ApJ 345, 245

Casewell, S. L., Dobbie, P. D., Hodgkin, S. T., et al. 2007, MNRAS, 378, 1131

Chabrier, G., Baraffe, I., Allard, F., \& Hauschildt, P. 2000, ApJ, 542, 464

Chambers, J. E. 2001, Icarus, 152, 205

Chen, C. H., Patten, B. M., Werner, M. W., et al. 2005, ApJ, 634, 1372

Cousins, A. W. J. 1976, Mem. R. Astr. Soc. 81, 25

Currie, T., Kenyon, S. J., Rieke, J., et al. 2007, ApJ, 663, L105

Cutri, R. M., Skrutskie, M. F., Van Dyk, S., et al. 2003, Explanatory Supplement to the 2MASS All Sky Data Release

Decin, G., Dominik, C., Waters, L. B. F. M., \& Waelkens, C. 2003, ApJ, 598, 636

Dobbie, P. D., Pinfield, D. J., Jameson, R. F., \& Hodgkin, S. T. 2002, MNRAS, 335, L79

Dodd, R. J. 2004, MNRAS, 355, 959

Dominik, C., \& Decin, G. 2003, ApJ, 598, 626

XMM-Newton Users Handbook 2008, ed. M. Ehle, I. de la Calle, Díaz Trigo, et al.

Engelbracht, C. W., Blaylock, M., Su, K. Y. L., et al. 2007, PASP, 119, 994

Evans, II N. J., \& the c2d Team 2003, PASP, 115, 965

Fazio, G. G., Hora, J. L., Allen, L. E., et al. 2004, ApJS, 154, 10

Forbes, M. C., Dodd, R. J., \& Sullivan, D. J. 2001, Baltic Astron., 10, 375

Fontanot, F., Somerville, R. S., \& Jester, S. 2007 [arXiv: 0711.1440]

Gautier, T. N. III, Rieke, G. H., Stansberry, J., et al. 2007, ApJ, 667, 527

Greene, T. P., Wilking, B. A., Andre, P., et al. 1994, ApJ, 434, 614

Gutermuth, R. A., Megeath, S. T., Muzerolle, J., et al. 2004, ApJS, 154, 374

Gutermuth, R. A., Myers, P. C., Megeath, S. T., et al. 2008, ApJ, 674, 336

Habing, H. J., Dominik, C., Jourdain de Muizon, M., et al. 2001, A\&A, 365, 545

Haish, K. E., Lada, E. A., \& Lada, C. J. 2001, ApJ, 553, 153

Harvey, P., Merín, B., Huard, T. L., et al. 2007, ApJ, 663, 1149
Hauschildt, P. H., Allard, F., \& Baron, E. 1999, ApJ, 512, 377

Hillenbrand, L. A. 2008, to appear in Physica Scripta - Special Issue on Nobel Symposium 135: Physics of Planetary Systems [arXiv:0805.0386]

Hog, E., Kuzmin, A., Bastian, U., et al. 1998, A\&A, 335, L65

Jameson, R. F., Dobbie, P. D., Pinfield, D. J., \& Hodgkin, S. T. 2003, in ed. E. L. Martìn (San Francisco: ASP), IAU Symp., 211, 171

Jeffries, R. D., Naylor, T., Devey, C. R., \& Totten, E. J. 2004, MNRAS, 351, 1401

Johnson, H. L. 1965, ApJ, 141, 936

Kessler-Silacci, J., Augereau, J.-C., Dullemond, C. P., et al. 2006, ApJ, 639, 275 Kharchenko, N. V. 2001, KFNT, 17, 409

Kharchenko, N. V., Piskunov, A. E., Roeser, S., et al. 2005, A\&A, 438, 1163

Kleine, T., Munker, C., Mezger, K., \& Palme, H. 2002, Nature, 418, 952

Lada, C. J. 1987, IAUS, 115, 1

Lada, C. J., Muench, A. A., Luhman, K. L., et al. 2006, AJ, 131, 1574

Landolt, A. U. 1992, AJ, 104, 340

Leggett, S. 1992, ApJS, 82, 351

Lodieu, N. 2005, AN, 326, 10, 1001

Lodieu, N., McCaughrean, M. J., Barrado Y Navascués, D., et al. 2005, A\&A, 436, 853

Lodieu, N., Hambly, N. C., Jameson, R. F., et al. 2006a, MNRAS, 374, 372

Lodieu, N., Hambly, N. C., \& Jameson, R. F. 2006b, MNRAS, 373, 95

Lodieu, N., Bouvier, J., James, D. J., et al. 2006c, A\&A, 450, 147

Lodieu, N., Dobbie, P. D., Deacon, N. R., et al. 2007, MNRAS, 380, 712

Luhman, K. L. 2004, ApJ, 616, 1033

Luhman, K. L., Whitney, B. A., Meade, M. R., et al. 2006, ApJ 647, 1280

Marino, A., Micela, G., Peres, G., et al. 2005, A\&A, 430, 287

Merín, B., Jorgensen, J., Spezzi, L., et al. 2008, ApJ, in press [arXiv: 0803.1504]

Mermilliod, J. C. 1981, A\&A, 97, 2351

Monet, D., Levine, S. E., Casian, B., et al. 2003, AJ, 125, 984

Moraux, E., Bouvier, J., Stauffer, J. R., \& Cullindre, J. C. 2003, A\&A, 400, 891

Muench, A. A., Lada, E. A., Lada, C. J., et al. 2003, ApJ, 125, 2029

Muench, A. A., Lada, C. J., Luhman, K. L., et al. 2007, AJ, 134, 411

Patten, B. M., \& Simon, T. 1996, ApJS, 106, 489

Patten, B. M., \& Pavlovski, C. M. 1999, PASP, 111, 210

Platais, I., Melo, C., Mermilliod, J.-C., et al. 2007, A\&A, 461, 509

Reach, W. T., Megeath, S. T., Cohen, M., et al. 2005, PASP, 117, 978

Reid, I. N. 2005, ASSL, 327, 53

Reipurth, B., \& Zinnecker, H. 1993, A\&A, 278, 81

Riaz, B., Mullan, D. J., \& Gizis, J. E. 2006, ApJ, 650, 1133

Richards, G. T., Croom, S. M., Anderson, S. F., et al. 2005, MNRAS, 360, 839

Robin, A. C., Reylé, C., Derriére, S., \& Picaud, S. 2003, A\&A, 409, 523

Robitaille, T. P., Whitney, B. A., Indebetow, R., et al. 2006, ApJS, 167, 256

Rolleston, W. R. J., \& Byrne, P. B. 1997, A\&AS, 126, 357

Sagar, R., \& Bhatt, H. C. 1989, MNRAS, 236, 865

Savage, B. D., \& Mathis, J. S. 1979, ARA\&A, 17, 73

Scholz, A., Jayawardhana, R., Wood, K., et al. 2007, ApJ, 660, 1517

Sicilia-Aguilar, A., Hartmann, L., Calvet, N., et al. 2006, ApJ, 638, 897

Sicilia-Aguilar, A., Hartmann, L. W., Watson, D., et al. 2007, ApJ, 659, 1637

Siegler, N., Muzerolle, J., Young, E. T., et al. 2007, ApJ, 654, 580

Simon, T., \& Patten, B. 1998, PASP, 110, 283

Spezzi, L., Alcalá, J. M., Frasca, A., et al. 2007, A\&A, 470, 281

Stauffer, J. R., Schultz, G., \& Kirkpatrick, J. D. 1998, ApJ, 499, L199

Stetson, P. B. 1987, PASP, 99, 191

The Guide Star Catalogue, Version 2.3.2, Space Telescope Science Institute (STScI) and Osservatorio Astronomico di Torino

Weingartner, J. C., \& Draine, B. T. 2001, ApJ, 548, 296

Young, E. T., Lada, C. J., Teixeira, P., et al. 2004, ApJS, 154, 428

Zacharias, N., Monet, D. G., Levine, S. E., et al. 2005, A\&AS, 205, 4815 